\title{
Les publications récentes (2006-2008) sur la préhistoire
}

Marie-Dominique Pinel et Catherine Le Gall

\section{(2) OpenEdition}

12 Journals

Édition électronique

URL : http://journals.openedition.org/rao/936

DOI : $10.4000 /$ rao.936

ISBN : 978-2-7535-1609-0

ISSN : $1775-3732$

Éditeur

Presses universitaires de Rennes

\section{Édition imprimée}

Date de publication : 31 décembre 2009

Pagination : 291-309

ISBN : 978-2-7535-1086-9

ISSN : 0767-709X

\section{Référence électronique}

Marie-Dominique Pinel et Catherine Le Gall, « Les publications récentes (2006-2008) sur la

préhistoire », Revue archéologique de l'Ouest [En ligne], 26 | 2009, mis en ligne le 31 décembre 2011, consulté le 06 décembre 2020. URL : http://journals.openedition.org/rao/936 ; DOI : https://doi.org/

$10.4000 /$ rao.936

Ce document a été généré automatiquement le 6 décembre 2020.

Tous droits réservés 


\title{
Les publications récentes (2006-2008) sur la préhistoire
}

\author{
Marie-Dominique Pinel et Catherine Le Gall
}

\section{Généralités}

1 Anonyme, 2008 - Petite histoire de l'archéologie en Centre-ouest Bretagne. Kreiz Breizh, 15, p. 14-19.

2 AQUILINA, M., 2008 - Recherches sur les associations de protection du patrimoine dans les villes du Morbihan : leur action hier et aujourd'hui. Bulletin et Mémoires de la Société polymathique du Morbihan, 134, p. 505-530.

3 AUBERT, G., CROIX, A., DENIS, M. et VEILLARD, J.-Y. (dir.), 2006 - Histoire de Rennes. Rennes, co-éd. Apogée - Presses universitaires de Rennes, 295 p.

4 BIROCHEAU, P., 2007 - Reportage sur le moulage d'un menhir de l'alignement du Douet à Hoedic (Morbihan). Bulletin du Groupe vendéen d'études préhistoriques, 43, p. 10-20.

5 BIZIEN-JAGLIN, C., 2006 - Chronique de prospection archéologique dans le nord de la Haute-Bretagne en 2005. Les dossiers du CeRAA, 34, p. 29-48.

BIZIEN-JAGLIN, C., 2007 - Chronique de prospection archéologique dans le nord de la Haute-Bretagne en 2006. Les dossiers du CeRAA, 35, p. 31-52.

7 BIZIEN-JAGLIN, C., 2008 - Chronique de prospection archéologique dans le nord de la Haute-Bretagne en 2007. Les dossiers du CeRAA, 36, p. 21-43.

BOUILLON, C. et CASSEN, S., 2008 - De l'Airbus A380 au site néolithique de la Table des Marchands (Morbihan): limites et perspectives d'un transfert de technologie, in VERGNIEUX, R. et DELEVOIE, C. (dir.) - Virtual Retrospect 2005 [proceedings of the conference, Biarritz (France), november 8th-10th 2005]. Bordeaux, Ausonius (Archéovision, 2), p. 157-164.

9 CASSARD, J.-C., CROIX, A., LE QUEAU, J.-R. et VEILLARD, J.-Y. (dir.), 2008 - Dictionnaire d'histoire de Bretagne. Morlaix, Skol Vreizh, $938 \mathrm{p}$. 
CERCAMON, C., 2008 - Marthe et Saint-Just Péquart, archéologues des îles du sud de la Bretagne (1923-1934). L'Archéologue - Archéologie nouvelle, $\mathrm{n}^{\circ}$ 98, p. 44-47.

CHARTIER, E., 2006 - Vingt ans d'archéologie. Une nouvelle approche de l'histoire de Bretagne. ArMen, $\mathrm{n}^{\circ} 151$, p. 28-37.

CHARTIER, E., 2008 - Une pirogue du Néolithique à Gavrinis. ArMen, nº 165, p. 50-51.

CHAURIS, L., 2008 - Extractions littorales de granite à Hoedic. Melvan - La revue des deux îles, 5, p. 115-125.

Collectif, 2006 - La mer pour mémoire. Archéologie sous-marine des épaves atlantique (catalogue d'exposition). Paris, Somogy, $368 \mathrm{p}$.

Collectif, 2007 - Marthe et Saint-Just Péquart, archéologues des îles. De Houat à Hoedic, 1923-1934. Melvan - La revue des deux îles, 4 ( $n^{\circ}$ spécial), 285 p.

CORRE, A., 2008 - Prospection inventaire en forêt de Rennes (Ille-et-Vilaine). Les dossiers du CeRAA, 36, p. 45-64.

DAIRE, M.-Y., 2006 - Îles et littoral de la Manche et de l'Atlantique. Chronique bibliographique 2003-2006. Bulletin d'information de l'AMARAI, 19, p. 115-127.

DAIRE, M.-Y., 2007 - Îles et littoral de la Manche et de l'Atlantique. Chronique bibliographique 2004-2007. Bulletin d'information de l'AMARAI, 20, p. 85-96.

DAIRE, M.-Y., 2008 - Îles et littoral de la Manche et de l'Atlantique. Chronique bibliographique 2005-2008. Bulletin d'information de l'AMARAI, 21, p. 111-1118.

DAVID, O., HINGUANT, S., MARCHAND, G., BOUJOT, C., TINEVEZ, J.-Y., GABILLOT, M., MENEZ, Y., MATTHIEU, N., MORICE, Y., BRANDHONNEUR, M., PICHOT, D., AUBERT, G., LAGADEC, Y., LETHUILLIER, J.-P., CAPDEVILLA, L. et SAINCLIVIER, J., 2006 - Bretagne est univers. Catalogue du Musée de Bretagne. Rennes, co-éd. Apogée - Presses universitaires de Rennes, $191 \mathrm{p}$.

GIRAUDON, D., 2007 - Le menhir de Saint-Uzec. Croyances populaires autour des mégalithes, ArMen, $\mathrm{n}^{\circ} 161$, p. 36-43.

LANGOUËT, L. et CHEVALIER, G., 2006 - Les cahiers de Victor Le Coniat. Apports à la Préhistoire de la région de Trégomar (Côtes-d'Armor). Les Dossiers du CeRAA, 34, p. 49-87

LARGE, J.-M., 2007 - Marthe et Saint-Just Péquart : un couple d'archéologues dans les îles du sud de la Bretagne, 1923-1934, in Marthe et Saint-Just Péquart, archéologues des îles. De Houat à Hoedic, 1923-1934. Melvan - La Revue des Deux Iles, 4 (nº spécial), p. 173-212.

2 LE CAM, G., 2006 - Zacharie Le Rouzic, enfant de Carnac, archéologue et... photographe. Bulletin de la Société d'Archéologie et d'Histoire du Pays de Lorient, 34 (2005-2006), p. 93-106.

LECERF, Y., 2008 - La construction rurale au Moyen Âge. Apport de l'archéologie expérimentale, in TONNERRE, N.-Y. (dir.) - La maison paysanne en Bretagne: 2500 ans d'habitat rural. Spézet, Coop Breizh, p. 36-43.

LEGENDRE, J.-P., OLIVIER, L. et SCHNITZLER, B., 2007 - Quand l'archéologie était au service du nazisme. Archéologia, n 442, p. 42-57.

LE NEZET, T., 2008 - La lente destruction des alignements mégalithiques du Gueldro. Bulletin de la Société d'Archéologie et d'Histoire du Pays de Lorient, 36 (2007-2008), p. 27-30.

LEVASSEUR, O., 2006 - Histoire de l'huître en Bretagne. Morlaix, Skol Vreizh, 84 p. 

Malo : un état de la question, in DAIRE, M.-Y. et LANGOUËT, L. (dir.) - Les pêcheries de Bretagne: Archéologie et Histoire des pêcheries d'estran. Rennes, co-éd. CeRAA - AMARAI, p. 61-66.

LOPEZ-ROMERO GONZALEZ DE LA ALEJA, E. et LE GALL, C., 2008 - Le fonds documentaire de Geoffroy d'Ault du Mesnil (1842-1921) et la recherche préhistorique en France. Les dossiers du CeRAA, 36, p. 65-72.

MAHIEU, E. et MAHIEU, F., 2007 - Les témoins pétrifiés.évolution et traditions des mégalithes de Bretagne. Theix, Actilia Multimédia, 45 p.

MENS, E., 2006 - Technologie des mégalithes dans l'ouest de la France. Bulletin et Mémoires de la Société polymathique du Morbihan, 132, p. 7-18.

MOUSSET, G., 2006 - Pointe de Kerpenhir. Menace sur les Pierres Plates de Locmariaquer d'après les notes écrites de Pierre Godec. Bulletin de la Société d'Archéologie et d'Histoire du Pays de Lorient, 34 (2005-2006), p. 15-20.

PRIGENT, G., 2007 - Inventaire du patrimoine des communes littorales des Côtesd'Armor : littoralité d'usages et culture littorale. ARSSAT - Bulletin Annuel, 32, p. 7-12.

QUERRE, G., HERBAULT, F., CALLIGARO, T., 2008 - Transport of Neolithic variscites demonstrated by PIXE analysis. X-Ray Spectrometry, vol. 37, Issue 2, p. 116-120.

REVER, F., JAMAUX, T. et JAMAUX, A., 2007 - Les autels tauroboliques du Mont-Dol. Aux origines des observations archéologiques de Chateaubriand. Saint-Malo, Alfred Jamaux, 78 p.

ROBIN, J., 2008 - Les sites antiques, Côtes-d'Armor Magazine, n 69, p. 35-37.

ROBIN, J., 2008 - Fouilles en sites médiévaux. Côtes-d'Armor Magazine, n 70, p. 35-37.

SALAUN, A., 2007 - De l'utilité d'un site archéologique et de son patrimoine. Patrimoine Bulletin de la Société archéologique de Corseul, 21, p. 30-32.

TARRÊTE, J. et LE ROUX, C.-T. (dir), 2008 - Le Néolithique. Paris, co-éd. Picard Ministère de la Culture et de la Communcation (coll. Archéologie de la France), $423 \mathrm{p}$.

TONNERRE, N.-Y. (dir.), 2008 - La maison paysanne en Bretagne : 2500 ans d'habitat rural. Spézet, Coop Breizh, 255 p.

VIGIER, E., 2007 - Images du passé : Carnac vu par Zacharie Le Rouzic. L'Archéologue Archéologie nouvelle, $\mathrm{n}^{\circ}$ 91, p. 55-60.

VIGIER, E., 2007 - L'apport des Péquart au musée de Carnac - De la dispersion du matériel de Téviec et d'Hoedic. In, Collectif. Marthe et Saint-Just Péquart, archéologues des îles. De Houat à Hoedic, 1923-1934. Melvan, La Revue des Deux Iles, 4 ( $\mathrm{n}^{\circ}$ spécial), p. 275-282.

\section{Publications diachroniques}

BERGER, C. et LE DOARÉ, R., 2007 - Le port de Ploumanac'h. ARSSAT - Bulletin annuel, 32, p. $19-25$.

BIZIEN-JAGLIN, C., 2008 - Les pêcheries, écluses, parcs et bouchots de Erquy à Saint-

CHAIGNEAU, C., 2007 - Des Mégalithes et des hommes : aujourd'hui en Pays de Redon. Saint-Just, co-éd. Nature et Mégalithes - CPIE Val de Vilaine. 71 p.

CLÉMENT, J.-H., 2008 - Les anciennes pêcheries, de la pointe d'Hillion à la Pointe de Pléneuf (Côtes-d'Armor), in DAIRE, M.-Y. et LANGOUËT, L. (dir.) - Les pêcheries de 
Bretagne: Archéologie et Histoire des pêcheries d'estran. Rennes, co-éd. CeRAA - AMARAI, p. 67-96.

Collectif, 2006 - Journée "Civilisations atlantiques \& archéosciences», Rennes, 8 avril 2006 (résumés des communications). Rennes, CNRS, Université de Rennes I, Ministère de la Culture, Université de Rennes 2 et Université de Nantes, 70 p.

Collectif, 2007 - Journée "Civilisations atlantiques \& archéosciences ", Rennes, 17 mars 2007 (résumés des communications). Rennes, CNRS, Université de Rennes I, Ministère de la Culture, Université de Rennes 2 et Université de Nantes,56 p.

Collectif, 2008 - Journée du "CReAAH» Archéologie, Archéosciences, Histoire, Rennes, 24 mai 2008 (résumés des communications). Rennes, CNRS, Université de Rennes I, Ministère de la Culture, Université de Rennes 2 et Université de Nantes, 57 p.

DAIRE, M.-Y., QUERRE, G., 2006 - Iron age ceramics in Western France: a multidisciplinary approach, in GHEORGHIU, D. (dir.) - Ceramic studies Papers on the social and cultural signifiance of ceramics in Europe and Eurasia from prehistoric to historic times. Oxford, Archaeopress, (BAR International Series, 1553), p. 63-68

51 DAIRE, M.-Y. et LANGOUËT, L. (dir.), 2008 - Les pêcheries de Bretagne: Archéologie et Histoire des pêcheries d'estran. Saint-Malo et Rennes, co-éd. CeRAA - AMARAI, $144 \mathrm{p}$.

DAIRE, M.-Y., 2008 - Des pêcheries d'estran sur les côtes du Bas-Léon, in DAIRE, M.-Y. et LANGOUËT, L. (dir.) - Les pêcheries de Bretagne: Archéologie et Histoire des pêcheries d'estran. Rennes, co-éd. CeRAA - AMARAI, p. 113-121.

DAIRE, M.-Y. et LANGOUËT, L., 2008 - Sur la chronologie des pêcheries fixes : le point de vue de l'archéologie, in DAIRE, M.-Y. et LANGOUËT, L. (dir.) - Les pêcheries de Bretagne: Archéologie et Histoire des pêcheries d'estran. Rennes, co-éd. CeRAA - AMARAI, p. 51-60.

DREANO, Y., GIOVANNI, S., DIETSCH-SELLAMI, M.-F., DUPONT, C., GRUET, Y., HOGUIN, R., IHUEL, E., LEROY, A., MARCHAND, G., PAILLER, Y., SPARFEL, Y. et TRESSET, A., 2007 Le patrimoine archéologique de l'ille Béniguet (Le Conquet, Finistère). Bilan des recherches 2000-2007. Bulletin de la Société de Sciences naturelles de l'ouest de la France (nouvelle série), 29 (3), p. 161-172.

55 GABILLOT, M. et MENEZ, Y., 2006 - Les âges du Bronze et du Fer, in Bretagne est univers. Catalogue du Musée de Bretagne. Rennes, co-éd. Apogée/Presses universitaires de Rennes, p. 40-51.

GALLIOU, P., LE GOFFIC, M., COATIVY, Y., EVEILLARD, J.-Y. et MARTINEAU, J., 2006 Notices d'archéologie finistérienne (année 2006). Bulletin de la Société archéologique du Finistère, 135, p. 11-27.

57 GALLIOU, P., LE GOFFIC, M., COATIVY, Y., EVEILLARD, J.-Y., PERENNEC, R. et PEUZIAT, J., 2007 - Notices d'archéologie finistérienne (année 2007). Bulletin de la Société archéologique du Finistère, 136, p. 11-36.

GAUTHIER, C., 2006 - Le patrimoine archéologique de l'estuaire de la Vilaine, du Néolithique à la période gallo-romaine. L'occupation humaine protohistorique. Bulletin d'information de l'AMARAI, 19, p. 41-56.

GUYODO, J.-N., 2007 - Installations néolithiques et gauloises à Er Yoc'h (Houat, Morbihan) : état de la question, in Collectif, Marthe et Saint-Just Péquart, archéologues des îles. De Houat à Hoedic, 1923-1934. Melvan, La Revue des Deux Iles, 4 ( $\mathrm{n}^{\circ}$ spécial), p. 229-250. 
, G., DAIRE, M.-Y., BARON, A., BAUDRY, A., DEFAIX, J., DESSE, J., DUPONT, C., GUYODO, J.-N. et VISSAC, C., 2006 - Peuplements préhistoriques et protohistoriques de l'ïle aux Moutons (Fouesnant, Finistère). Journée "Civilisations atlantiques \& archéosciences", Rennes, 8 avril 2006 (résumés des communications). Rennes, CNRS, Université de Rennes I, Ministère de la Culture, Université de Rennes 2 et Université de Nantes, p. 26-27.

HINGUANT, S., MARCHAND, G., BOUJOT, C. et TINEVEZ, J.-Y., 2006 - Du Paléolithique au Néolithique, in Bretagne est univers. Catalogue du Musée de Bretagne. Rennes, co-éd. Apogée - Presses universitaires de Rennes, p. 23-39.

LANGOUET, L., DAIRE, M.-Y. et QUESNEL, L., 2008 - Les pêcheries maritimes et estuariennes du Morbihan : première approche interdisciplinaire, in DAIRE, M.-Y. et LANGOUËT, L. (dir.) - Les pêcheries de Bretagne: Archéologie et Histoire des pêcheries d'estran. Rennes, co-éd. CeRAA - AMARAI, p. 123-143.

LARGE, J.-M. et LE BANNIER, J.-C., 2006 - Une archéologie de l'île de Houat (Morbihan). Inventaire des sites pré- et protohistoriques. Bulletin d'information de l'AMARAI, 19, p. 5-40.

64 LARGE, J.-M., 2006 - L'alignement du Douet à Hoedic : suite de l'opération de fouille programmée 2005 (Morbihan). Étude de l'occupation pré- et protohistorique. Journée "Civilisations atlantiques \& archéosciences", Rennes, 8 avril 2006 (résumés des communications). Rennes, CNRS, Université de Rennes I, Ministère de la Culture, Université de Rennes 2 et Université de Nantes, p. 34-35.

LARGE, J.-M., 2006 - Opération de prospection-inventaire à Houat en 2005. Étude de l'occupation pré- et protohistorique. Journée "Civilisations atlantiques \& archéosciences", Rennes, 8 avril 2006 (résumés des communications). Rennes, CNRS, Université de Rennes I, Ministère de la Culture, Université de Rennes 2 et Université de Nantes, p. 33.

66 LE BIHAN, J.-P., 2007 - Quimper-Locmaria antique. Une genèse et une chronologie encore complexes. Archéopages, 20, p. 38-43.

67 LE BIHAN, J.-P., 2007 - Les cultes antiques à Ouessant. Deux millénaires d'étranges pratiques religieuses. ArMen, $n^{\circ}$ 160, p. 6-15.

68 LE BIHAN, J.-P., 2007 - Du Bronze moyen à l'aube du Moyen Âge, un lieu de culte révélateur de la fonction insulaire atlantique : Ouessant - Mez Notariou. AREMORICA. études sur l'ouest de la Gaule romaine, 1, p. 15-37.

LE BIHAN, J.-P., GUILLAUMET, J.-P., MENIEL, P., ROUSSOT-LARROQUE, J. et VILLARD, J.F., 2007 - Du Bronze moyen à l'Antiquité, un lieu de culte inscrit dans la longue durée : Mez-Notariou - Ouessant, in BARRAL, P., DAUBIGNEY, A., DUNNING, C. et al. - L'âge du Fer dans l'arc jurassien et ses marges. Dépôts, lieux sacrés et territorialité à l'âge du Fer, volume 2.(29e colloque international de l'AFEAF, Bienne, 5-8 mai 2005). Besançon, Presses universitaires de Franche-Comté, p. 629-652.

70 LE GOFFIC, M., 2006 - Aperçu de la préhistoire et de l'archéologie du Cap Sizun, Bulletin de l'Association bretonne, 115 p. 43-60.

71 LE PENNEC, C., 2006 - Découvertes archéologiques récentes au centre-ville de Vannes. Bulletin et Mémoires de la Société polymathique du Morbihan, 132, p. 69-87.

72

LE PERSON, A., 2007 - Les installations portuaires de Lannion. ARSSAT - Bulletin Annuel, 32, p. 14-17.

Revue archéologique de l'Ouest, 26 | 2009 
MENIEL, P., 2008 - Approche taphonomique le dépôt d'Ouessant « Mez Notariou », in MENIEL, P. - Manuel d'archéozoologie funéraire et sacrificielle. Gollion (Suisse), éd. Infolio, p. 117-122.

MEURET, J.-C., 2006 - Visseiche, vingt-cinq siècles d'aristocratie, in De l'archéologie à l'écrit., Mémoires de la Société d'Histoire et d'Archéologie de Bretagne, 84 (Congrès de Vitré, 8-10 septembre 2005), p. 131-180.

MORICE, Y., BRANDHONNEUR, M. et PICHOT, D., 2006 - Du Paléolithique au Néolithique, in Bretagne est univers. Catalogue du Musée de Bretagne. Rennes, Apogée, Presses Universitaires de Rennes, p. 72-93.

MOUSSET, G., 2006 - Rivière d'Etel : découverte fortuite d'un site archéologique. Bulletin de la Société d'Archéologie et d'Histoire du Pays de Lorient, 34 (2005-2006), p. 23-26.

NAUDINOT, N., 2008 - Les armatures lithiques tardiglaciaires dans l'ouest de la France (Régions Bretagne et Pays de la Loire) : Proposition d'organisation chrono-culturelle et chaîne opératoire de fabrication, in PETILLON, J.-M., DIAS-MEIRINHO, M.-H. CATTELAIN, P., HONEGGER, M., NORMAND, C. et VALDEYRON, N. (coord.) - Recherches sur les armatures de projectiles du Paléolithique supérieur au Néolithique (actes du colloque C83, XVe congrès de l'UISPP, Lisbonne, 4-9 septembre 2006), Palethnologie, 1, p. 250-277.

8 PRIGENT, G., 2008 - Histoire et ethnologie des pêcheries entre Trieux et Arguenon, in DAIRE, M.-Y. et LANGOUËT, L. (dir.) - Les pêcheries de Bretagne : Archéologie et Histoire des pêcheries d'estran. Rennes, co-éd. CeRAA - AMARAI, p. 97-112.

ROUÉ, D et LE GOFF, J.-C., 2008 - Barrages à poissons et pêcheries à Santec (NordFinistère), in DAIRE, M.-Y. et LANGOUËT, L. (dir.) - Les pêcheries de Bretagne : Archéologie et Histoire des pêcheries d'estran. Rennes, co-éd. CeRAA - AMARAI, p. 97-111.

0 SALLIER-DUPIN, G. de, 2007 - Sur les traces de mégalithes et autres sites préhistoriques oubliés dans les Côtes-d'Armor. Les Dossiers du CeRAA, 35, p. 53-71.

81 TINEVEZ, J.-Y., 2006 - L'éperon barré de La Rochette, Mauron (Morbihan). Journée "Civilisations atlantiques \& archéosciences", Rennes, 8 avril 2006 (résumés des communications). Rennes, CNRS, Université de Rennes I, Ministère de la Culture, Université de Rennes 2 et Université de Nantes, p. 10-12.

2 TINEVEZ, J.-Y. et QUESNEL, L., 2008 - Mauron, La Rochette. Multiples occupations d'un promontoire. Fouille programmée 2003-2006 (Morbihan). Journée du "CReAAH» Archéologie, Archéosciences, Histoire, Rennes, 24 mai 2008 (résumés des communications). Rennes, CNRS, Université de Rennes I, Ministère de la Culture, Université de Rennes 2 et Université de Nantes, p. 26-27.

\section{Paléoenvironnement et archéologie du paysage}

83 ANTOINE, A. et MARGUERIE, D. (dir.), 2007 - Bocages et Sociétés. (Colloque du CERHIO et du CREAAH, Université de Rennes 2, 29 septembre-1er octobre 2004). Rennes, Presses universitaires de Rennes (coll. Espace et Territoires), 509 p.

ANTOINE, A., MARGUERIE, D., MARCHAND, J.-P., BAUDRY, J. et BUREL, F., 2007 - Trente ans après... in ANTOINE, A. et MARGUERIE, D. (dir.) - Bocages et sociétés, (Colloque du CERHIO et du CREAAH, Université de Rennes 2, 29 septembre-1er octobre 2004). Rennes, Presses universitaires de Rennes (coll. Espace et Territoires), p. 9-21. 
AOUSTIN, D. et BATT, M., 2008 - L'impact d'un habitat du Moyen-Âge sur le paysage : l'exemple du site archéologique du Goënidou dans les monts d'Arrée, In La lande. Un paysage au gré des hommes (colloque International de Châteaulin, 15-17 février 2007), Brest, CRBC, p. 45-56.

BARDEL, P., MAILLARD, J.-L. et PICHARD, G., 2008 - L'arbre et la haie : mémoire et avenir du bocage. Rennes, co-éd. Ecomusée du pays de Rennes - Presses universitaires de Rennes, 191 p. + 1 DVD.

7 BAUDRY, A. et DAIRE, M.-Y., 2006 - Premières approches archéozoologiques sur le site de Port-Blanc à Hoedic. "Civilisations atlantiques \& archéosciences", Rennes, 8 avril 2006 (résumés des communications). Rennes, CNRS, Université de Rennes I, Ministère de la Culture, Université de Rennes 2 et Université de Nantes, p. 41-42.

8 BERNARD, V., RENAUDIN, S. et MARGUERIE, D., 2006 - Evidence of trimmed oaks (Quercus sp.) in North Western France during the early Middle Ages (9th-11th centuries A.D.), in DUFRAISSE, A. (éd.) - Charcoal analysis: new analytical tools and methods for archaeology. Oxford, Archaeopress, (BAR International Series, 1483), p. 103-108

9 BERNARD, V., EPAUD, F. et LE DIGOL, Y., 2007 - Bois de haie, bois de bocage, bois d'architecture, in ANTOINE, A. et MARGUERIE, D. (dir.) - Bocages et Sociétés. (Colloque du CERHIO et du CREAAH, Université de Rennes 2, 29 septembre-1er octobre 2004). Rennes, Presses universitaires de Rennes (coll. Espace et Territoires), p. 213-230.

B BLAIN, S., PLANCHON, O., BERNARD, V., 2006 - Cartographie des données dendrochronologiques du nord-ouest de la France pour la période médiévale (XIVe-XVe siècles), in Les risques liés au temps et au climat: actes du 19e colloque de l'Association internationale de climatologie (AIC), Épernay, France, 6 - 9 septembre 2006, p. 131-135.

1 BOCHER, E., PENVEN, M.-J. et BEDEL, O., 2007 - Organisation spatiale des systèmes bocagers et parcours des écoulements de surface, in ANTOINE, A. et MARGUERIE, D. (dir.) - Bocages et Sociétés. (Colloque du CERHIO et du CREAAH, Université de Rennes 2, 29 septembre-1er octobre 2004). Rennes, Presses universitaires de Rennes (coll. Espace et Territoires), p. 245-260 $+24 \mathrm{pl}$.

CATTEDDU, I. et MARGUERIE, D., 2007 - Une création parcellaire carolingienne : les établissements agricoles médiévaux de Montours (Ille-et-Vilaine), in ANTOINE, A. et MARGUERIE, D. (dir.) - Bocages et Sociétés. (Colloque du CERHIO et du CREAAH, Université de Rennes 2, 29 septembre-1er octobre 2004). Rennes, Presses universitaires de Rennes (coll. Espace et Territoires), p. 81-90.

DIETRICH, A., 2008 - Les bois travaillés, in LE CLOIREC, G. - Carhaix antique : la domus $d u$ Centre hospitalier. Contribution à l'histoire de Vorgium, chef-lieu de la cité des Osismes. Rennes, Presses Universitaires de Rennes, 2008, p. 151-158.

DIETSCH-SELLAMI, M.-F., 2007 - L'utilisation des plantes sauvages au Néolithique dans le nord-ouest de la France : témoignages carpologiques, in BESSE, M. (dir.) -Sociétés néolithiques. Des faits archéologiques aux fonctionnements socio-économiques.(27e colloque interrégional sur le Néolithique, Neuchâtel, 1-2 octobre 2005). Cahiers d'Archéologie romande, 108, p. 127-136.

DIETSCH-SELLAMI, M.-F., 2008 - étude carpologique de deux puits du site gallo-romain du campus de la place Hoche à Rennes, in POUILLE, D. - Rennes antique. Rennes, Presses universitaires de Rennes, p. 391-398. 

p. 34-35. Paris, éd. du CTHS, p. 295-309. Archaeobotany, 17 ( $\mathrm{n}^{\circ}$ 5), p. 585-596.

DUPONT, C., 2006 - La malacofaune de sites mésolithiques et néolithiques de la façade atlantique de la France. Contribution à l'économie et à l'identité culturelle des groupes concernés. Oxford, Archaeopress (BAR International Series, 1571), 438 p.

DUPONT, C., 2007 - Les amas coquilliers mésolithiques de Téviec et d'Hoedic et le dépôt coquillier néolithique d'Er Yoc'h: de la ressource alimentaire à l'utilisation des coquilles vides, in Marthe et Saint-Just Péquart, archéologues des îles. De Houat à Hoedic, 1923-1934. Melvan - La Revue des Deux Îles, 4 (nº spécial), p. 251-264.

DUPONT, C., JEAN, F., PAULET, Y.-M. et QUERNE, J., 2008 - Quand la patelle nous livres ses secrets : la stratégie de collecte des coquillages du site archéologique de Beg-arLoued (île de Molène, Finistère). Journée du "CReAAH» Archéologie, Archéosciences, Histoire, Rennes, 24 mai 2008 (résumés des communications). Rennes, CNRS, Université de Rennes I, Ministère de la Culture, Université de Rennes 2 et Université de Nantes,

DUPONT, C., ÁLVAREZ-FERNÁNDEZ, E. et GRUET, Y., 2008 - Un nouveau crustacé identifié sur le site gaulois de Port Blanc (île d'Hoedic, Morbihan): le pouce-pied Pollicipes pollicipes (Gmelin, 1790). Bulletin de l'AMARAI, 21, p. 17-23.

GABILLOT, M., GAUDIN, L., MARGUERIE, D., MARCOUX, N. et BERNARD, V., 2007 Indicateurs d'activités agro-pastorales et métallurgiques dans le Massif armoricain au cours du deuxième millénaire avant notre ère, in RICHARD, H., MAGNY, M. et MORDANT, C. (dir.) - Environnements et cultures à l'Âge du Bronze en Europe occidentale.

GAUDIN, L., MARGUERIE, D. et MARCOUX, N., 2007 - étude palynologique de la tourbière de Langazel en Trémaouezan (Finistère), in DURFORT, J. (dir.) - Les Tourbières de Bretagne. Mèze, éd. Biotope, (Les cahiers du naturalistes de Bretagne), p. 107-109.

GAUDIN, L., MARGUERIE, D. et LANOS, P., 2008 - Correlation between spatial distributions of pollen data, archaeological records and physical parameters from north-western France: a GIS and numerical analysis approach. Vegetation History and

GEBHARDT, A., 2007 - Impacts anthropiques anciens sur les sols forestiers. Quelques études de cas en contexte archéologique et expérimental, in La mémoire des forêts. (Colloque "Forêt, archéologie et environnement », Velaine-en-Haye, 14-16 décembre 2004). Paris, co-éd. ONF - INRA - DRAC de Lorraine, p. 211-218.

GEBHARDT, A., MARGUERIE, D., VISSET, L., BERNARD, V. et GAUDIN, L., 2007 - Des premiers agriculteurs aux bocages armoricains, les données des disciplines paléoenvironnementales, in ANTOINE, A. et MARGUERIE, D. (dir.) - Bocages et Sociétés. (Colloque du CERHIO et du CREAAH, Université de Rennes 2, 29 septembre-1er octobre 2004). Rennes, Presses universitaires de Rennes (coll. Espace et Territoires), p. 51-61.

5 JOLY, C., DELALANDE, C. et VISSET, L., 2008 - De la possible présence d'arbres à Hoedic... (Morbihan, Bretagne). Journée du «CReAAH» Archéologie, Archéosciences, Histoire, Rennes, 24 mai 2008 (résumés des communications). Rennes, CNRS, Université de Rennes I, Ministère de la Culture, Université de Rennes 2 et Université de Nantes, p. 20-23.

6 LE DU-BLAYO, L. et ROUSSEAU, P., 2007 - Des politiques d'arasement aux politiques de replantation : le cas du rebocagement en Bretagne, in ANTOINE, A. et MARGUERIE, D. (dir.) - Bocages et Sociétés. (Colloque du CERHIO et du CREAAH, Université de Rennes 2, 
29 septembre-1er octobre 2004). Rennes, Presses universitaires de Rennes (coll. Espace et Territoires), p. 163-174.

LE GOFFIC, M., 2007 - Archéologie et tourbières de Bretagne, in DURFORT, J. (dir.) - Les Tourbières de Bretagne. Mèze, éd. Biotope (Les cahiers du naturalistes de Bretagne), p. 109-112.

108 LEMOUlAND, Q. et PERRIN, G., 2007 - Impact de l'habitat du Bas Moyen Âge du Goënidou et de son parcellaire associé sur l'environnement actuel : approche par une étude de végétation (Berrien, 29), in La mémoire des forêts. (colloque " Forêt, archéologie et environnement », Velaine-en-Haye, 14-16 décembre 2004). Paris, co-éd. ONF - INRA DRAC de Lorraine, p. 94-93.

LOPEZ-ROMERO GONZALEZ DE LA ALEJA, E., 2008 - Characterizing the evolution of visual landscapes in the late prehistory of south-west morbihan (Brittany, France). Oxford Journal of Archaeology, 27 (3), p. 217-239.

110 MARGUERIE, D., 2007 - Palynologie et tourbières en Bretagne, in DURFORT, J. (dir.) - Les Tourbières de Bretagne, Mèze, éd. Biotope (Les cahiers du naturalistes de Bretagne), p. 101-106.

111 MARGUERIE, D., 2007 - Mutations paysagères sur le temps long : l'exemple de Montours (Ille-et-Vilaine), in LE DU-BLAYO, L. - Le paysage en Bretagne. Quimper, éd. Palantines, p. 220-221.

112 MARGUERIE, D. et OILLIC, J.-C., 2007 - Pollens et haies du bocage dans le nord-ouest de la France, in ANTOINE, A. et MARGUERIE, D. (dir.) - Bocages et Sociétés. (colloque du CERHIO et du CREAAH, Université de Rennes 2, 29 septembre-1er octobre 2004). Rennes, Presses universitaires de Rennes (coll. Espace et Territoires), p. 105-119.

113 MARGUERIE, D. et HUNOT, J.-Y., 2007 - Charcoal analysis and dendrology: data from archaeological sites in Western France. Journal of Archaeological Sciences, 34, p. 1417-1433.

114 MARGUERIE, D., 2008 - Histoire des essences et des usages en Bretagne. Des millénaires d'interaction entre végétation et agriculteurs bretons, les données de l'archéobotanique, in BARDEL, P., MAILLARD, J.-L. et PICHARD, G. (dir.) - L'arbre et la haie, mémoire et avenir du bocage. Rennes, co-éd. écomusée du pays de Rennes - Presses universitaires de Rennes, p. 92-95.

115 MEURET, J.-C. et LE MOULAND, Q., 2007 - Parcellaires de manoirs et origines du bocage de l'ouest de la France : le cas de La Montagne à Visseiche (Ille-et-Vilaine), in ANTOINE, A. et MARGUERIE, D. (dir.) - Bocages et Sociétés. (colloque du CERHIO et du CREAAH, Université de Rennes 2, 29 septembre-1er octobre 2004). Rennes, Presses universitaires de Rennes (coll. Espace et Territoires), p. 91-104.

PERICHON, S., 2006 - Les noms de lieux signalant des bois, des landes, des haies et des essences bocagères en Ille-et-Vilaine. Annales de Bretagne et des Pays de l'Ouest, 113 (1), p. 7-23.

VISSET, L. et BERNARD, J., 2006 - évolution du littoral et du paysage, de la presqu'île de Rhuys à la rivière d'Etel (Massif armoricain - France), du Néolithique au Moyen Âge. Archéosciences, 30, p. 143-156.

VISSET, L., DELALANDE, C. et JOLY, C., 2006 - Tourbière et marécage à Hoedic. Histoire du paysage depuis 6000 ans. Melvan - La Revue des deux îles, 3, p. 25-35. 


\section{Paléolithique - Mésolithique}

BLANCHET, S., KAYSER, O., MARCHAND, G. et YVEN, E., 2006 - Le Mésolithique moyen en Finistère : de nouvelles datations pour le groupe de Bertheaume. Bulletin de la Société préhistorique française, 103, p. 507-517.

COSTA, L.-J. et MARCHAND, G., 2006 - Transformation des productions lithiques du premier au second Mésolithique en Bretagne et en Irlande. Bulletin de la Société préhistorique française, 103 (2), p. 275-290.

GALLUDEC, M., 2006 - Présence de galets aménagés sur le littoral de Ploemeur (56). Bulletin de la Société d'Archéologie et d'Histoire du Pays de Lorient, 34 (2005-2006), p. 5-10.

GALLUDEC, M. et LE GUEN, A., 2007 - Un biface triangulaire à talon cortical. Bulletin de la Société d'Archéologie et d'Histoire du Pays de Lorient, 35 (2006-2007), p. 13-16.

GALLUDEC, M., 2008 - De nouveaux galets aménagés sur le littoral de Ploemeur (56): quatre choppers en quartzite. Bulletin de la Société d'Archéologie et d'Histoire du Pays de Lorient, 36 (2007-2008), p. 13-15.

HUET B., 2007 - Matières premières et comportements techno-économiques au Paléolithique moyen dans le Massif armoricain : le cas des industries à composante lithologique mixte. Journée "Civilisations atlantiques \& archéosciences", Rennes, 17 mars 2007 (résumés des communications). Rennes, CNRS, Université de Rennes I, Ministère de la Culture, Université de Rennes 2 et Université de Nantes, p. 45.

LAFORGE, M., MONNIER, J.-L. et HALLEGOUET, B., 2008 - Contribution to the chronostratigraphy of the Lower Palaeolithic site of Menez-Dregan 1 (Plouhinec, Finistère, France). Correlations with Gwendrez cliff Pleistocene deposits, inQuaternary Stratigraphy between Atlantic and Continental Europe (Colloque international INQUA/SEQS, Rennes, 22-27 septembre 2008), Conference Abstracts, p. 27. LARGE, J.-M., 2008 - Pointes de flèche à Houat. La lettre de Melvan, n 11, p. 3.

LEFORT, J.-P., MARCOUX, N. et MONNIER, J.-L., 2007 - Apport de la géologie marine à la détermination des sources de matières premières au Paléolithique dans le Massif armoricain: origine possible du silex utilisé sur les stations de Menez-Dregan (Finistère, France). Implications paléoclimatiques et paléoenvironnementales. Journée "Civilisations atlantiques \& archéosciences", Rennes, 17 mars 2007 (résumés des communications). Rennes, CNRS, Université de Rennes I, Ministère de la Culture, Université de Rennes 2 et Université de Nantes, p. 46-48.

LEFORT, J.-P., MARCOUX, N. et MONNIER, J.-L., 2007 - Apport de la géologie marine à la détermination des sources de matières premières au Paléolithique dans le Massif armoricain: origine possible du silex utilisé sur les stations de Menez-Dregan (Finistère, Finistère, France). Implications paléoclimatiques et paléoenvironnementales. Quaternaire, 18 (3), p. 233-241.

129 LE GUEN, A., 2007 - «Le Gorzed », une station du Mésolithique moyen sur le côte occidentale de l'île de Groix. Bulletin de la Société d'Archéologie et d'Histoire du Pays de Lorient, 35 (2006-2007), p. 27-37.

130 MARCH R. J., MONNIER J.-L. et LARGEAU, C., 2006 - L'homo erectus et le feu: la géochimie sur la trace des plus anciens foyers européens, in Les matières organiques en France : état de l'art et prospectives (actes du colloque de Carqueranne, 22-24 janvier 2006). Carqueranne, co-éd. CNRS - INRA - INSU - IHSS, p. 50. 
MARCHAND, G., 2007 - Et maintenant, qu'est-ce qu'on fait ? Le Mésolithique de l'Ouest, 80 ans après M. et St.-J. Péquart, in Marthe et Saint-Just Péquart, archéologues des îles. De Houat à Hoedic, 1923-1934. Melvan, La Revue des Deux Iles, 4 (nº spécial), p. 213-228.

MARCHAND, G. et TSOBGOU-AHOUPE, R., 2007 - Comprendre la diffusion des roches au Mésolithique en Bretagne : analyse structurale des matériaux et variabilité technique. Archéosciences, 31, p. 113-126.

MOLINES, N., 2007 - Paléogéographie, matières premières et territoires exploitables dans l'ouest de la France au Pléistocène moyen, in MONCEL, M.-H., MOIGNE, A.-M., ARZARELLO, M. et al. - Aires d'approvisionnement en matières premières et aires d'approvisionnement en ressources alimentaires. Approche intégrée des comportements. Oxford, Archaeopress - UISPP, p. 61-71.

MONNIER, J.-L., 2006 - Les premiers peuplements de l'ouest de la France. Cadre chronostratigraphique et paléoenvironnemental. Bulletin du Musée d'Anthropologie préhistorique de Monaco, 46, p. 3-20.

MONNIER, J.-L. et MOLINES, N., 2007 - Le Paléolithique armoricain et l'essor de la préhistoire en France, in EVIN, J. (dir.) - Un siècle de construction du discours scientifique en Préhistoire, volume $I$ « Des idées d'hier... ». (26 ${ }^{\mathrm{e}}$ congrès préhistorique de France - Congrès du Centenaire, Avignon-Bonnieux, 21-25 septembre 2004). Paris, SPF, p. 351-357.

MONNIER, J.-L., HALLEGOUET, B. et HUET, B., 2008 - Menez-Dregan à Plouhinec ou les premiers habitants de l'extrême ouest de l'Eurasie. Bulletin municipal de Plouhinec, p. 2-3.

MONNIER, J.-L. et HUET, B., 2008 - Sedimentological applications to correlate eroded palaeolithic layers with local Pleistocene stratigraphy. A contribution to geological dating, inQuaternary Stratigraphy between Atlantic and Continental Europe (Colloque international INQUA/SEQS, Rennes, 22-27 septembre 2008), Conference Abstracts, p. 37-38.

PAILLER, Y. et DUPONT, C., 2007 - Analyse fonctionnelle des galets biseautés du Mésolithique à la fin du Néolithique dans l'ouest de la France, l'ouest des îles britanniques, Bulletin de la Société préhistorique française, 104 (1), p. 31-54.

TSOBGOU-AHOUPE, R., 2006 - Chimie, structure de la matière et modalités du débitage des ultramylonites et cataclasites armoricaines : exemple du site mésolithique final de Creac'h Miné Vihan (France) Archéosciences, 30, p. 109-118.

YVEN, E., 2006 - La simplification de l'outillage lithique à la fin du Mésolithique moyen en Bretagne occidentale : une adaptation à la partition des territoires, in ASTRUC, L., BON, F., LEA, V., MILCENT, P.-Y. et PHILIBERT, S. (dir.) - Normes techniques et pratiques sociales. De la simplicité des outillages pré- et protohis-toriques, (26e rencontres internationales d'archéologie et d'histoire d'Antibes, 20-22 octobre 2005). Antibes, APDCA, p. 135-145.

\section{Néolithique}

Anonyme, 2006 - Archéologie : étonnantes découvertes au Douet (Hoedic). La lettre de Melvan, $\mathrm{n}^{\circ}$ 6, p. 2 . 

- Archéologie nouvelle, $n^{\circ}$ 90, p. 49-50. Archéologie nouvelle, $\mathrm{n}^{\circ}$ 98, p. 52. Morbihan). Internéo, 6, p. 77-86. universitaires de Rennes, p. 33-54. (Archéovision, 2), p. 187-193. 49, p. 197-258. Nantes, $71 \mathrm{p}$. $n^{\circ} 283$, p. 16-17.

Anonyme, 2007 - L'alignement de la Dame du Douet à Hoedic (Morbihan). L'Archéologue

Anonyme, 2007 - Une carrière de menhirs? à La Trinité-sur-Mer (Morbihan). L'Archéologue - Archéologie nouvelle, $\mathrm{n}^{\circ}$ 92, p. 52.

Anonyme, 2008 - Haches polies, presqu'île de Quiberon (Morbihan). L'Archéologue -

BOUJOT, C. et PINET, L., 2007 - Mégalithes et pierres dressées, matériau du discours scientifique en Préhistoire : évolution et perspectives d'après les exemples de Carnac (Bretagne) et du plateau de Cauria (Corse), in EVIN, J. (dir.) - Un siècle de construction du discours scientifique en Préhistoire, volume III "...Aux conceptions d'aujourd'hui» (26e congrès préhistorique de France - Congrès du Centenaire, Avignon-Bonnieux, 21-25 septembre 2004). Paris, SPF, p. 185-197.

CASSEN, S., 2006 - From underground to extramound: recognition and interpretation of funerary barrows in southern Armorica (France, Vth millennium BC), in SMEJDA, L., Turek, J. \& THRANE, H. (ed.) - Archaeology of burial mounds (EAA 9th annual meeting, Saint-Petersburg 2003). Plzen, University of West Bohemia, p. 22-37.

CASSEN, S. et FRANÇOIS, P., 2006 - Du Chasséen armoricain à l'Auzay-Sandun : un apport de l'ACR 2003-2006 sur le site de la Table des Marchand (Locmariaquer,

CASSEN, S., LABRIFFE, P.-A. de et MÉNANTEAU, L., 2006 - Le sel « chauffé » des baies marines en Armorique-sud durant les Ve et IVe millénaires avant J.-C. : à la recherche (ouest-européenne) de croyances et de faits techniques, in HOCQUET, J.-C. et SARRAZIN, J.-L. - Le sel de la baie. Histoire, archéologie, ethnologie des sels atlantiques. Rennes, Presses

CASSEN, S., ROBIN, G., LEFÈBVRE, B. et MERHEB, M., 2006 - Moving the immovable. A short study of methods of recording and illustrating Neolithic engraved steles in Brittany, in VERGNIEUX, R. et DELEVOIE, C. (dir.) - Virtual Retrospect 2005 (proceedings of the conference, Biarritz (France), november 8th-10th 2005). Bordeaux, Ausonius,

CASSEN, S., 2007 - Le Mané Lud en images. Interprétations de signes gravés sur les parois de la tombe à couloir néolithique de Locmariaquer (Morbihan). Gallia-Préhistoire,

CASSEN, S., 2007 - Un pour tous, tous contre un... Symboles, mythe et histoire à travers une stèle morbihannaise du Ve millénaire, in TESTART, A., BARRAY, L. et BRUN, P. (dir.) - Pratiques funéraires et sociétés. Nouvelles approches en archéologie et en anthropologie sociale (actes du colloque interdisciplinaire de Sens, 12-14 juin 2003). Dijon, Presses universitaires de Dijon (Art, Archéologie et patrimoine), p. 37-67.

CASSEN, S. (dir.), 2007 - Autour de la Table. Explorations archéologiques et discours savants sur une architecture néolithique restaurée à Locmariaquer, Morbihan (pré-actes du colloque international, Vannes, 5-7 oct. 2007), co-éd. Université de Bretagne-sud - Université de

CASSEN, S. et PÉTREQUIN, P., 2008 - Pietre sotto il cielo e asce sotto il mare. Archeo,

CASSEN, S., LABRIFFE, P.-A. de et MÉNANTEAU, L., 2008 - Washing and heating on the neolithic shores of Western Europe. An archaeological hypothesis on the production of 
sea salt, in WELLER, O., DUFRAISSE, A et PÉTREQUIN, P. (dir.) - Sel, eau et forêt, hier et aujourd'hui. Besançon, Presses universitaires de Franche-Comté, p. 175-204.

CASSEN, S., BOUJOT, C., ERRERA, M., MARGUERIE, D., MENIER, D., PAILlER, Y., PÉTREQUIN, P., POIRIER, S., VEYRAT, E. et VIGIER, E., 2008 - Discovery of an underwater deposit of Neolithic polished axeheads and a submerged stone alignment at Petit Rohu near Saint-Pierre-Quiberon (Morbihan, France). Antiquity, 83 ( $\mathrm{n}^{\circ} 316$ ) [en ligne; consulté le 22 juillet 2009 - Disponible sur http://www.antiquity.ac.uk/ProjGall/ cassen/index.html].

CHEVALIER, G., 2007 - Une hache naviforme en méta-hornblendite à l'Armor-Pleubian (Côtes-d'Armor). Bulletin d'information de l'AMARAI, 20, p. 67-72.

DREANO, Y., GIOVANNACCI, S., DUPONT, C., GRUET, Y., HOGUIN, R., IHUEL, E., LEROY, A., MARCHAND, G., PAILLER, Y., SPARFEL, Y. et TRESSET, A. 2007 - Le patrimoine archéologique de l'île Béniguet (Le Conquet, Finistère) - Bilan des recherches 2000-2007, in Quinze ans d'étude et de recherches sur la réserve de Béniguet, Bulletin de la Société des Sciences naturelles de l'ouest de la France, nouvelle série, 29 (3), p. 161-172. ERRERA, M., PÉTREQUIN, P., PÉTREQUIN, A.- M., CASSEN, S. et CROUTSCH, C., 2007 Contribution de la spectroradiométrie à la compréhension des transferts longuedistance des lames de hache au Néolithique. Bulletin de la Société tournaisienne de Géologie, Préhistoire et Archéologie, 10 (4), p. 101-142. FROMONT, N., HUARD, J., KERDIVEL, G., LERIVRAIN, R., MENS, E., et QUERRE, G., 2008 Sites néolithiques d'acquisition et de transformation de la dolérite en Mayenne. Journée du «CReAAH» Archéologie, Archéosciences, Histoire, Rennes, 24 mai 2008 (résumés des communications). Rennes, CNRS, Université de Rennes I, Ministère de la Culture, Université de Rennes 2 et Université de Nantes, p. 30-33.

161 GALLUDEC, M. et LE GUEN, A., 2007 - Découverte de deux haches polies, à Larmor-Plage et Guidel. Bulletin de la Société d'Archéologie et d'Histoire du Pays de Lorient, 35 (2006-2007), p. 25-26.

162 GAUMÉ, E., 2007 - Cornes d'aurochs (supplique pour le réexamen d'une gravure néolithique de bovidé dans l'île morbihannaise de Gavrinis, Bretagne). Bulletin de la Société préhistorique française, 104 (1), p. 81-88. GOUÉZIN, P., 2007 - Les mégalithes du Morbihan littoral. Au sud des Landes de Lanvaux, de Guidel à Quiberon. Rennes, co-éd. ICB - CeRAA, (coll. Patrimoine archéologique de Bretagne), $134 \mathrm{p}$.

HINGUANT, S., 2007 - Un ensemble mégalithique exceptionnellement conservé. Archéopages, $\mathrm{n}^{\circ}$ 18, p. 70-71.

HINGUANT, S., GUYODO, J.-N. et HAMON, G. 2007 - Une occupation littorale campaniforme à Saint-Malo (Ille-et-Vilaine), in Camps, enceintes et structures d'habitat néolithiques en France septentrionale.(24e colloque interrégional sur le Néolithique, Orléans, 19-21 novembre 1999). Revue archéologique du Centre de la France, suppl. 27, p. 309-325.

166 HOGUIN, R., PAILLER, Y. et GIOVANNACCI, S., 2006 - Étude de quelques stations néolithiques de surface repérées sur l'île Béniguet (Le Conquet, Finistère). Bulletin d'information de l'AMARAI, 19, p. 57-68.

167 JOUSSAUME, R. et LAPORTE, L., 2006 - Monuments funéraires néolithiques dans l'ouest de la France, in JOUSSAUME, R., LAPORTE, L. et SCARRE, C. (dir.) - Origine et 
développement du mégalithisme de l'ouest de l'Europe (colloque international, Musée des Tumulus de Bougon, 26-30 octobre 2002). Bougon, co-éd. Conseil général des DeuxSèvres - Musée des Tumulus de Bougon, p. 319-343.

LANGOUËT, L. (et coll.), 2006 - Les mégalithes de l'arrondissement de Guingamp. Rennes, coéd. ICB - CeRAA (coll. Patrimoine archéologique de Bretagne), $92 \mathrm{p}$.

LANGOUËT, L., 2006 - Quelques réflexions sur les mégalithes des Côtes-d'Armor. Les Dossiers du CeRAA, 34, p. 89-102.

170 LANGOUËT, L., GOUÉZIN, P., BIHAN, S., LOPEZ-ROMERO, E., 2007 - Louis Le Pontois et les monuments disparus ou détériorés de la région de Lorient. Les Dossiers du CeRAA, 35, p. 5-30.

171 LANGOUËT, L., GOUÉZIN, P., BIHAN, S. et LOPEZ-ROMERO, E., 2007 - Louis Le Pontois et les monuments mégalithiques de l'île de Groix (Morbihan). Bulletin d'information de l'AMARAI, 20, p. 5-33.

172 LAPORTE, L., GUYODO, J.-N. et BIZIEN-JAGLIN, C., 2007 - Nouvelles découvertes en milieu humide autour de l'habitat ceinturé du Néolithique moyen à Lillemer (Ille-etVilaine, France), in BESSE, M. (dir.) - Sociétés néolithiques. Des faits archéologiques aux fonctionnements socio-économiques(27e colloque Interrégional sur le Néolithique, Neuchâtel, 1-2 octobre 2005). Cahiers d'Archéologie romande, 108, p. 341-349.

LARGE, J.-M., MENS, E. et DELOZE, V., 2007 - L'alignement du Douet à Hoedic (Morbihan). Journée "Civilisations atlantiques \& archéosciences", Rennes, 17 mars 2007 (résumés des communications). Rennes, CNRS, Université de Rennes I, Ministère de la Culture, Université de Rennes 2 et Université de Nantes, p. 36-37.

174 LARGE, J.-M., 2007 - Nouveaux sondages archéologiques au Groah Denne et au Lan Vihan (Hoedic). La lettre de Melvan, juin 2007, p. 4-5.

175 LARGE, J.-M., 2008 - Campagne de prospection thématique Hoedic 2007 : Groah Denn et Lann Vihan. Journée du "CReAAH » Archéologie, Archéosciences, Histoire, Rennes, 24 mai 2008 (résumés des communications). Rennes, CNRS, Université de Rennes I, Ministère de la Culture, Université de Rennes 2 et Université de Nantes, p. 20-23.

176 LARGE, J.-M., 2008 - Des pierres qui parlent : l'apport de la fouille de l'alignement du Douet à Hoedic (Morbihan). Melvan - La Revue des Deux Iles, 5, p. 9-28.

177 LARGE, J.-M. et MENS, E., 2008 - L'alignement du Douet à Hoedic (Morbihan, France). L'anthropologie, 112, p. 544-571.

LE COLLETER, C., 2007 - Préhistoire de Lanester : le dolmen de Malachappe. Bulletin de la Société d'Archéologie et d'Histoire du Pays de Lorient, 35 (2006-2007), p. 17-23.

LECORNEC, J., 2007 - Naviguer en Atlantique au Néolithique. Bulletin et Mémoires de la Société polymathique du Morbihan, 133, p. 13-26.

180 LE GOFFIC, M., 2006 - La nécropole mégalithique de la pointe du Souc'h en Plouhinec (Finistère). Journée "Civilisations atlantiques \& archéosciences", Rennes, 8 avril 2006 (résumés des communications). Rennes, CNRS, Université de Rennes I, Ministère de la Culture, Université de Rennes 2 et Université de Nantes, p. 24-25.

181 LE GUEN, A., 2008 - Néolithique moyen : expansion chaséenne aux abords du ruisseau de Pont-Christ. Brech (56). Bulletin de la Société d'Archéologie et d'Histoire du Pays de Lorient, 36 (2007-2008), p. 33-42. 
LE QUELLEC, J.-L., 2006 - L'art mégalithique en France : récents développements, in JOUSSAUME, R., LAPORTE, L. et SCARRE, C. (dir.) - Origine et développement du mégalithisme de l'ouest de l'Europe (colloque international, Musée des Tumulus de Bougon, 26-30 octobre 2002). Bougon, co-éd. Conseil général des Deux-Sèvres - Musée des Tumulus de Bougon, p. 687-717.

LE ROUX, C.-T., 2006 - Pierres dressées dans l'ouest de la France, in JOUSSAUME, R., LAPORTE, L. et SCARRE, C. (dir.) - Origine et développement du mégalithisme de l'ouest de l'Europe (colloque international, Musée des Tumulus de Bougon, 26-30 octobre 2002). Bougon, co-éd. Conseil général des Deux-Sèvres - Musée des Tumulus de Bougon, p. 545-565.

184 LE ROUX, C.-T. (dir.) - Monuments mégalithiques à Locmariaquer (Morbihan). Le long tumulus d'Er Grah dans son environnement. Gallia-Préhistoire, suppl. 38, 308 p. Gisserot, $112 \mathrm{p}$.

LE ROUX, C.-T., 2007 - Gavrinis face aux temps et aux hommes. Bulletin et Mémoires de la Société polymathique du Morbihan, 133, p. 7-12. LOPEZ-ROMERO GONZALEZ DE LA ALEJA, E., 2007 - Analyse territoriale des premières sociétés agro-pastorales en Bretagne: premiers résultats pour la région Blavet-Laïta. Journée "Civilisations atlantiques \& archéosciences", Rennes, 17 mars 2007 (résumés des communications). Rennes, CNRS, Université de Rennes I, Ministère de la Culture, Université de Rennes 2 et Université de Nantes, p. 34-35.

LOPEZ-ROMERO GONZALEZ DE LA ALEJA, E., 2008 - Monuments néolithiques de la région de Lorient (Morbihan, Bretagne) : à propos des modes d'organisation des territoires. L'Anthropologie, 112 (4-5), p. 572-597.

MARCHAND, G., PAILLER, Y. et TOURNAY, G., 2006 - Carrément à l'Ouest! Indices du Villeneuve-Saint-Germain au centre de la Bretagne (Le Dillien à Cléguérec et Bellevue à Neulliac, Morbihan). Bulletin de la Société préhistorique française, 103 (3), p. 519-533. MARCHAND, G., GUYODO, J.-N., HAMON, G. et PAILLER, Y., 2007 - Le Néolithique ancien de l'ouest de la France: nouvelles données en Bretagne et dans les îles anglonormandes. Journée "Civilisations atlantiques \& archéosciences", Rennes, 17 mars 2007 (résumés des communications). Rennes, CNRS, Université de Rennes I, Ministère de la Culture, Université de Rennes 2 et Université de Nantes, p. 38-39.

191 MARCHAND, G., 2007 - Neolithic fragrances: Mesolithic-Neolithic interactions in western France. In WHITTLE, A. (dir.), CUMMINGS, V. (dir.), Going Over: The MesolithicNeolithic Transition in North-West Europe. Londres, British Academy, (Proceedings of the British Academy, 144), p. 225-242.

192 MENS, E., 2006 - Méthodologie de l'étude technologique des gravures néolithiques armoricaines (France), in JOUSSAUME, R., LAPORTE, L. et SCARRE, C. (dir.) - Origine et développement du mégalithisme de l'ouest de l'Europe (colloque international, Musée des Tumulus de Bougon, 26-30 octobre 2002). Bougon, co-éd. Conseil général des DeuxSèvres - Musée des Tumulus de Bougon, p. 719-725.

193 MENS, E., 2006 - Technologie des mégalithes dans l'ouest de la France. Remontage mental de l'affleurement et chronologie technique du débitage. Bulletin et Mémoires de la Société polymathique du Morbihan, 132, p. 7-18. 

p. 353-359. des Tumulus de Bougon, p. 369-373. Series, 1648), $340 \mathrm{p}$. p. 163-177. 2005). Antibes, APDCA, p. 419-433.

MENS, E., 2007 - Étude technologique des mégalithes de l'ouest de la France, les monuments néolithiques du Mané-Bras et du Mané-Bihan à Locoal-Mendon (Morbihan), in EVIN, J. (dir.) - Un siècle de construction du discours scientifique en Préhistoire, volume III "...Aux conceptions d'aujourd'hui» (26e congrès préhistorique de France - Congrès du Centenaire, Avignon-Bonnieux, 21-25 septembre 2004). Paris, SPF,

PAILLER, Y. et SIMON, J.-M., 2006 - Découverte de deux lames polies en fibrolite à Carentoir (Morbihan). Les dossiers du CeRAA, 34, p. 5-7.

PAILLER, Y. et SPARFEL, Y., 2006 - La nécropole mégalithique de Zouliérou (Ile Molène, Finistère), in JOUSSAUME, R., LAPORTE, L. et SCARRE, C. (dir.) - Origine et développement du mégalithisme de l'ouest de l'Europe (colloque international, Musée des Tumulus de Bougon, 26-30 octobre 2002). Bougon, co-éd. Conseil général des Deux-Sèvres - Musée

PAILLER, Y., 2007 - Des dernières industries à trapèzes à l'affirmation du Néolithique en Bretagne occidentale (5500-3500 avant J.-C.). Oxford, Archaeopress, (BAR International

PAILLER Y., MARCHAND G., BLANCHET S., GUYODO J.-N. et HAMON G., 2008 - Le Villeneuve-Saint-Germain dans la péninsule Armoricaine : les débuts d'une enquête, in BURNEZ-LANOTTE, L., ILETT, M. et ALLARD, P. (dir.) - Fin des traditions danubiennes dans le Néolithique du Bassin parisien et de la Belgique (5100-4700 av. J.-C.). Autour des recherches de Claude Constantin. Paris, co-éd. Société préhistorique française - Presses universitaires de Namur, (Mémoires de la Société préhistorique française, 44), p. 91-111.

PÉTREQUIN, P., CASSEN, S. etCROUTSCH, C., 2006 - Imitation ou convergence : les haches néolithiques à talon perforé au nord-ouest des Alpes, in Artisanats, sociétés et civilisations. Hommage à J.-P. Thévenot. Revue archéologique de l'Est, suppl. 24,

PÉTREQUiN, P., PÉTREQUIN, A.-M., CASSEN, S., CROUTSCH, C. et ERRERA, M., 2006 Complexité technique et valorisation sociale : haches polies de Nouvelle-Guinée et du Néolithique alpin, in ASTRUC, L., BON, F., LEA, V., MILCENT, P.-Y. et PHILIBERT, S. (dir.) - Normes techniques et pratiques sociales. De la simplicité des outillages pré- et protohistoriques (26e Rencontres internationales d'archéologie et d'histoire d'Antibes, 20-22 octobre

PÉTREQUIN, P., PÉTREQUIN, A.-M., ERRERA, M., CASSEN, S., CROUTSCH, C., DUFRAISSE, A., GAUTHIER, E. et ROSSY M., 2007 - Les carrières néolithiques du Monviso (Piémont, Italie). Chronologie et conditions d'exploitation, in La pierre en milieu alpin, de la Préhistoire au Moyen-Âge: exploitation, utilisation et diffusion (XIe colloque international sur les Alpes dans l'antiquité, Bagnes/Champsec, 15-17 sept. 2006), Bulletin d'études préhistoriques et archéologiques alpines, 18, p. 168-188.

PÉTREQUIN, P., PÉTREQUIN, A.-M., ERRERA, M., CROUTSCH, C., CASSEN, S. et ROSSY M., 2007 - Les carrières néolithiques de jadéitite du Monviso (Piémont, Italie) : un premier survol, in BESSE, M. (dir.) - Sociétés néolithiques. Des faits archéologiques aux fonctionnements socio-économiques (colloque interrégional sur le Néolithique, 1-2 octobre 2005, Neuchâtel), Cahiers d'Archéologie romande, 108, p. 51-68.

PÉTREQUIN, P., SHERIDAN, A., CASSEN, S., ERRERA, M., GAUTHIER, E., KLASSEN, L., LE MAUX, N. et PAILLER, Y., 2008 - Neolithic Alpine axeheads, from the Continent to Great Britain, the Isle of Man and Ireland. Analecta Praehistorica Leidensia, 40, p. 261-279. 
204

Fer: du bon usage des collections anciennes. Journée du «CReAAH» Archéologie, Archéosciences, Histoire, Rennes, 24 mai 2008 (résumés des communications). Rennes, CNRS, Université de Rennes I, Ministère de la Culture, Université de Rennes 2 et Université de Nantes, p. 45-47.

Revue archéologique de l'Ouest, 26 | 2009 
LE GOFFIC, M., 2007 - Implantation géographique et topographique des sépultures de l'âge du bronze dans le Finistère, in BURGESS, C. (ed.) - Beyond Stonehenge, essays on the Bronze Age in honour of Colin Burgess, Oxford, Oxbow books, p. 57-64.

LE GOFFIC, M. et NALLIER, R., 2008 - Fouille d'un caveau du Bronze ancien à Lannilis (Finistère). Journée du «CReAAH » Archéologie, Archéosciences, Histoire, Rennes, 24 mai 2008 (résumés des communications). Rennes, CNRS, Université de Rennes I, Ministère de la Culture, Université de Rennes 2 et Université de Nantes, p. 41-43.

217 NALLIER, R. et LE GOFFIC, M., 2008 - Rosnoën 60 ans après. Compléments et révision concernant le dépôt de l'âge du Bronze final de Penavern (Finistère). Bulletin de la Société préhistorique française, 105 (1), p. 131-157.

218 SERVAT, J., 2008 - L'habitat de l'âge du Bronze de Leslouc'h, à Plouedern (Finistère). L'Archéologue - Archéologie nouvelle, n 97, p. 57.

\section{7. Âge du Fer}

ABOLLIVIER, P., 2008 - Numismatique et archéologie en Armorique occidentale à la fin de l'âge du Fer. Le monnayage des Osismes. St Germain en Laye, éd. Commios, 352 p.

ABOLLIVIER, P., 2008 - Catalogue des monnaies gauloises du Musée départemental breton, Quimper (Finistère). Bulletin de la Société archéologique du Finistère, 136, p. 37-58.

Anonyme, 2007 - Des enceintes protohistoriques à Lamballe (Côtes-d'Armor) L'Archéologue - Archéologie nouvelle, n 87, p. 48.

Anonyme, 2008 - Le Port-Blanc, sur l'île de Hoedic (Morbihan). L'Archéologue Archéologie nouvelle, $\mathrm{n}^{\circ}$ 94, p. 53-54.

BAUDRY, A. et DAIRE, M.-Y., 2006 - Premières approches archéozoologiques : PortBlanc, île de Hoedic (Morbihan). Journée "Civilisations atlantiques \& archéosciences", Rennes, 8 avril 2006 (résumés des communications). Rennes, CNRS, Université de Rennes I, Ministère de la Culture, Université de Rennes 2 et Université de Nantes, p. 41-42.

BLONDEL, F., 2006 - Étude comparative réalisée à partir des sanctuaires gaulois à l'époque de La Tène (480 à 30 avant notre ère). Bulletin de l'APAREA, 3, p. 15-24.

CHARTIER, E., 2008 - L'or des Osismes. ArMen, n 163, p. 58-59.

CHARTIER, E., 2008 - Une ferme gauloise à Vitré. ArMen, nº 164, p. 58.

DAIRE, M.-Y., BAUDRY, A. et QUESNEL, L., 2006 - Le site gaulois de Port-Blanc à Hoedic (Morbihan). La campagne de fouille de 2005, premiers résultats. Bulletin d'information de l'AMARAI, 19, p. 69-86.

DAIRE, M.-Y., BAUDRY, A., LEVILLAYER, A. et QUESNEL, L., 2006 - Du sel gaulois sur l'île de Groix (Morbihan). Bilan des sondages à Locmaria - Pointe des Saisies (2003-2006). La campagne de fouille de 2005, premiers résultats. Bulletin d'information de l'AMARAI, 20, p. 47-65.

DAIRE, M.-Y. et QUESNEL, L., 2007 - Du sel gaulois sur l'île de Groix (Morbihan). Bilan des sondages 2004-2006. Journée "Civilisations atlantiques \& archéosciences", Rennes, 17 mars 2007 (résumés des communications). Rennes, CNRS, Université de Rennes I, Ministère de la Culture, Université de Rennes 2 et Université de Nantes, p. 18-20.

DAIRE, M.-Y., 2007 - Plaidoyer pour un outillage lithique de l'Âge du Fer: le cas des maillets à gorge de l'ouest de la France. L'Anthropologie, 111, p. 106-131. 

p. 53-56. archéologique de l'Ouest, 25, p. 93-137. Nouvelle, n' 97, p. 20-22. Archéologie nouvelle, $n^{\circ}$ 97, p. 53-54. nouvelle, $\mathrm{n}^{\circ}$ 97, p. 14-17. archéologique de Corseul, 22, p. 6-8.

DAIRE, M.-Y., 2007 - La production de sel dans les îles et sur le littoral breton à l'époque gauloise. Bulletin de la Société d'Archéologie et d'Histoire du Pays de Lorient, 35 (2006-2007),

DAIRE, M.-Y., 2008 - Des Gaulois sur l'île Guennoc (Landéda, Finistère). Revue

DAIRE, M.-Y. et QUESNEL, L., 2008 - L'île Guennoc et son occupation gauloise, 40 ans après (Landéda, Finistère). Journée du "CReAAH» Archéologie, Archéosciences, Histoire, Rennes, 24 mai 2008 (résumés des communications). Rennes, CNRS, Université de Rennes I, Ministère de la Culture, Université de Rennes 2 et Université de Nantes, p. 56-57.

DAIRE, M.-Y., 2008 - Sel et salaisons des Gaulois d'Armorique (Dossier «L'Armorique celtique, la Bretagne celtique, la Bretagne gauloise»). L'Archéologue - Archéologie

DAIRE, M.-Y., 2008 - Le Port-Blanc, sur l'île de Hoedic (Morbihan). L'Archéologue -

DAIRE, M.-Y., 2008 - Des stèles gauloises par milliers... L'Archéologue - Archéologie

DAIRE, M.-Y. et LOPEZ-ROMERO, E., 2008 - Les Gaulois de la côte... Syndrome du Coucou? (Dolmens et Gaulois). (Dossier « L'Armorique celtique, la Bretagne celtique, la Bretagne gauloise »). L'Archéologue - Archéologie nouvelle, n 97, p. 32-33.

DREANO, Y., 2008 - Etude de la vieille commune (Labrus bergylta) du sondage 2 de La Tène finale sur l'île aux Moutons, in BEAREZ, P., GROARD, S. et CLAVEL, B. (dir.) Archéologie du poisson. 30 ans d'archéo-ichtyologie au CNRS. Hommage aux travaux de JeanDesse et Nathalie Desse-Berset. ( $28^{\mathrm{e}}$ rencontres internationales d'archéologie et d'histoire d'Antibes), Antibes, APDCA, p. 201-205.

FISCHER, B., 2008 - Une étonnante monnaie coriosolite. Patrimoine, bulletin de la Société

FORRE, P. et JACQUET, H., 2006 - Découverte d'un four à sel à petits et gros piliers au sud du Morbihan sur la plage du Logui à Pénestin (Morbihan). Feuillets mensuels de la Société nantaise de Préhistoire, n 439, p. 44-47.

FRÈRE, D., 2008 - Les relations entre la Méditerranée et l'Armorique protohistorique. Patrimoine, bulletin de la Société archéologique de Corseul, 22, p. 15-23.

HUNTER, F., 2006 - Art in Later Iron Age society, in HASELGROVE, C. - Celtes et Gaulois. L'Archéologie face à l'histoire. Les mutations de la fin de l'âge du Fer. (Actes de la table ronde de Cambridge, 7-8 juillet 2005). Glux-en-Genne, Bibracte, Centre archéologique européen, (coll. Bibracte, 12/4), p. 93-115.

GOMEZ de SOTO, J., 2006 - L'art laténien du Ve et du IVe siècles avant J.-C. en Gaule de l'ouest. Monde nord-alpin et/ou méditerranée ? Actualités de l'art celtique d'Occident, in FRÈRE, D. (dir.) - De la Méditerranée vers l'Atlantique. Aspects des relations entre la Méditerranée et la Gaule centrale et occidentale (VIIle-Ile siècles avant J.-C.). Rennes, Presses universitaires de Rennes (coll. Archéologie \& Culture), p. 57-65.

GOMEZ de SOTO, J., 2007 - L'art celtique ancien (La Tène A-B, Ve-Ive siècles avant J.-C.) en Centre-Ouest : état de la question, in Sites et mobiliers de l'âge du Fer entre Loire et Dordogne. Chauvigny, Association des publications Chauvinoises, p. 9-11. 
GOMEZ de SOTO, J., 2007 - Le tumulus du Bonethève à Pressignac (Charente, France) et les tombes du premier et du début du second Âge du Fer à éléments d'équipement équestre ou pièces de char en Gaule occidentale. Archäologisches Korrespondenzblatt, 37, 2, p. 221-232.

GOMEZ de SOTO, J., 2007 - Tombes du premier et du début du second âge du Fer à éléments d'équipement équestre ou pièces de char en Gaule occidentale. Journée "Civilisations atlantiques \& archéosciences", Rennes, 17 mars 2007 (résumés des communications). Rennes, CNRS, Université de Rennes I, Ministère de la Culture, Université de Rennes 2 et Université de Nantes, p. 27-28.

HALDEMANN, M. et LE GOFF, I., 2007 - Étude d'une nécropole de la fin du premier âge du Fer en Bretagne : le cimetière de Boquidet à Sérent (Morbihan). Revue archéologique de l'Ouest, 24, p. 55-72.

A.-L., 2008 - La ferme gauloise de la Grande Haie à Vitré. (Dossier "L'Armorique celtique, la Bretagne celtique, la Bretagne gauloise »). L'Archéologue Archéologie nouvelle, $\mathrm{n}^{\circ}$ 97, p. 34.

249 LANGOUËT, L., 2008 - Oppida ou sites fortifiés de l'Ouest armoricain. (Dossier «L'Armorique celtique, la Bretagne celtique, la Bretagne gauloise »). L'Archéologue Archéologie nouvelle, $\mathrm{n}^{\circ}$ 97, p. 4-9.

250 LECORNEC, J., 2006 - Une particularité de l'âge du Fer armoricain: les souterrains. Bulletin et Mémoires de la Société polymathique du Morbihan, 132, p. 19-53.

LE GOFF, E., 2007 - Découverte d'un habitat groupé de la fin de la période gauloise chez les Osismes : nouvelles perspectives pour aborder l'occupation du sol et la structuration du territoire laténien du secteur de Quimper (Finistère). AREMORICA. Études sur l'ouest de la Gaule romaine, 1, p. 9-14.

LE GOFF, E., 2008 - L'agglomération gauloise de Kergolvez à Quimper. (Dossier "L'Armorique celtique, la Bretagne celtique, la Bretagne gauloise »). L'Archéologue Archéologie nouvelle, $\mathrm{n}^{\circ}$ 97, p. 23.

MALRAIN, F., 2007 - Habitats aristocratiques au second âge du Fer. Mise en évidence de la diversité des élites par la confrontation des vestiges archéologiques. Archéopages, $\mathrm{n}^{\circ} 19$, p. $18-25$.

MARTON, A., 2007 - Note sur une lampe «trouvée à Taden» (Côtes-d'Armor). Les Dossiers du CeRAA, 35, p. 81-83.

MENEZ, Y. et GOMEZ DE SOTO, J., 2006 - L'habitat de Kergariou à Quimper (Finistère). Journée "Civilisations atlantiques \& archéosciences", Rennes, 8 avril 2006 (résumés des communications). Rennes, CNRS, Université de Rennes I, Ministère de la Culture, Université de Rennes 2 et Université de Nantes, p. 28-29.

MENEZ, Y. et GOMEZ de SOTO, J., 2006 - L'habitat de Kergariou à Quimper. Bulletin de l'AFEAF, 24, p. 83-85.

MENEZ, Y., BERRANGER, M. et VILLARD-LE TIEC, A., 2006 - L'environnement de la forteresse aristocratique de Paule. Bilan des fouilles effectuées en 2005 (Côtesd'Armor). Journée "Civilisations atlantiques \& archéosciences ", Rennes, 8 avril 2006 (résumés des communications). Rennes, CNRS, Université de Rennes I, Ministère de la Culture, Université de Rennes 2 et Université de Nantes, p. 30-32.

MENEZ, Y. et BERRANGER, M., 2007 - Le site de Paule : nouvelles découvertes (Côtesd'Armor). Journée "Civilisations atlantiques \& archéosciences", Rennes, 17 mars 2007 
(résumés des communications). Rennes, CNRS, Université de Rennes I, Ministère de la Culture, Université de Rennes 2 et Université de Nantes, p. 16-17.

MENEZ, Y., VIVET, J.-B., CHANSON, K. et DUPRE, M., 2007 - La forge de Paule (Côtesd'Armor), in L'économie du fer protohistorique : de la production à la consommation du métal ( $28^{\mathrm{e}}$ colloque de l'AFEAF, Toulouse, 20-23 mai 2004). Aquitania, suppl. 14/2, p. 213-237.

MENEZ, Y., 2008 - Les bâtiments du second âge du Fer en Bretagne, in TONNERRE, N.-Y. (dir.) - La maison paysanne en Bretagne: 2500 ans d'habitat rural. Spézet, Coop Breizh, p. 10-15.

MEURET, J.-C., 2006 - Un dépôt de lingots de fer de type currency-bars: Le ClosMaugendre - Visseiche (Ille-et-Vilaine). Journée "Civilisations atlantiques \& archéosciences", Rennes, 8 avril 2006 (résumés des communications). Rennes, CNRS, Université de Rennes I, Ministère de la Culture, Université de Rennes 2 et Université de Nantes, p. 55-57.

262 MEURET, J.-C., 2007 - Une « ferme gauloise » : la Montagne - Visseiche (Ille-et-Vilaine). Journée "Civilisations atlantiques \& archéosciences", Rennes, 17 mars 2007 (résumés des communications). Rennes, CNRS, Université de Rennes I, Ministère de la Culture, Université de Rennes 2 et Université de Nantes, p. 21-22.

MORNAND, J., 2008 - Mise au point sur les enceintes et les cairns du Menez-Hom (Finistère) : enceintes Ar C'horn Tro, Menez-Hielc'h, Parc ar C'herc'h et cairns du Yed, du Yedig, à l'ouest d'Ar C'horn Tro, dit « Tombeau du roi Marc'h », Saint Nic. Bulletin de la Société archéologique du Finistère, 136, p. 59-63.

MOUSSET, G., 2006 - Kerhillio-Erdeven: territoire gaulois. Bulletin de la Société d'Archéologie et d'Histoire du Pays de Lorient, 34 (2005-2006), p. 21-22.

PIGEAUD, R., 2008 - Le trésor gaulois de Laniscat. Archéologia, n 451, p. 4-7.

SERVAT, J., 2008 - Un trésor gaulois retrouvé à Laniscat (Côtes-d'Armor). L'Archéologue Archéologie nouvelle, $\mathrm{n}^{\circ}$ 94, p. 51-52.

267 TANGUY, D., 2008 - Habitat aristocratique : Kerven-Teignouse à Inguiniel (Morbihan). (Dossier "L'Armorique celtique, la Bretagne celtique, la Bretagne gauloise»). L'Archéologue - Archéologie nouvelle, $\mathrm{n}^{\circ}$ 97, p. 10-13.

TANGUY, D., 2008 - L'habitat gaulois du Talhouet à Pluvigner (Morbihan). (Dossier "L'Armorique celtique, la Bretagne celtique, la Bretagne gauloise »). L'Archéologue Archéologie nouvelle, $\mathrm{n}^{\circ}$ 97, p. 18-19.

269 VILLARD, J.-F., LE BIHAN, J.-P., PLUTON, S. et GAUMÉ, E., 2006 - La nécropole à crémations du Hallstatt final - La Tène ancienne de Kerjaouen en Quimper (Finistère). Revue archéologique de l'Ouest, 23, p. 171-198.

VILLARD-LE TIEC A., LE DELLIOU G. et LORHO T., 2006 - Ploemeur «Kerham ». Un monument funéraire du premier âge du Fer? (Morbihan). Journée "Civilisations atlantiques \& archéosciences ", Rennes, 8 avril 2006 (résumés des communications). Rennes, CNRS, Université de Rennes I, Ministère de la Culture, Université de Rennes 2 et Université de Nantes, p. 50-51.

271 VILLARD-LE TIEC, A., 2007 - Les monuments circulaires du premier âge du Fer de Ploemeur « Kerham - Lann Porz Menec'h » et «Lann Tinikeï » (Morbihan). Bulletin de la Société d'Archéologie et d'Histoire du Pays de Lorient, 35 (2006-2007), p. 47-51. 
VILLARD-LE TIEC, A., 2007 - Ploemeur, Kerham - Lann-Porz-Menec'h (Morbihan) : un monument funéraire du premier âge du Fer ? Revue archéologique de l'Ouest, 24, p. 31-53.

VILLARD-LE TIEC, A., 2008 - Pratiques funéraires de l'extrême ouest de la Gaule. (Dossier «L'Armorique celtique, la Bretagne celtique, la Bretagne gauloise»). L'Archéologue - Archéologie nouvelle, $\mathrm{n}^{\circ}$ 97, p. 28-31.

VIVET, J.-B., 2007 - La production du fer protohistorique en haute Bretagne d'après les résultats des prospections, des fouilles d'ateliers et des analyses archéométriques, in L'économie du fer protohistorique : de la production à la consommation du métal. (28e colloque de l'AFEAF, Toulouse, 20-23 mai 2004). Aquitania, suppl. 14/2, p. 63-84.

\section{8. Époque gallo-romaine}

ALLONSIUS, C. et GROETEMBRIL, S., 2008 - Quelques exemples de restitutions basées sur l'étude des peintures murales. Archéopages, n 21, p. 67-71

AMANDRY, M. et HOLLARD, D., 2006 - Le trésor de Bourg-Blanc 1989 (Finistère), in Trésors de l'ouest de la France. Trésors monétaires, XXII. Paris, Bibliothèque Nationale de France,p. 119-132+ 2 pl.

Anonyme, 2006 - La villa gallo-romaine de Taden. Archéologia, n 431, p. 5.

Anonyme, 2006 - Fouilles archéologiques à Carhaix. La réserve des terrains Le Manac'h, campagnes 2000/2006. Rennes, co-éd. SRA de Bretagne - Conseil général du Finistère Ville de Carhaix-Plouguer, n. p.

Anonyme, 2006 - Nouvelles 2006: La Villa gallo-romaine des Alleux à Taden (Côtesd'Armor). Patrimoine, bulletin de la Société archéologique de Corseul, 20, p. 45.

Anonyme, 2007 - Une luxueuse bâtisse romaine se cachait sous la lande. Mané-Véchen : une villa venue d'ailleurs. Sciences Ouest, $n^{\circ}$ 248, p. 6-7.

Anonyme, 2008 - Vorgium, capitale gallo-romaine des Osismes. La réserve archéologique de Carhaix. Finistère Penn-ar-Bed, n 107, p. 42-43.

Anonyme, 2008 - Un centaure découvert à Châteaugiron, ArMen, n 167, p. 60.

Anonyme, 2008 - Monnaie romaine au Fort Bloqué. Bulletin du Comité d'Histoire du Pays de Ploemeur, $\mathrm{n}^{\circ} 18$, p. 48.

ARRAMOND, J.-C. et REQUI, C., 2006 - La villa gallo-romaine de la Gare au Quiou. Patrimoine, bulletin de la Société archéologique de Corseul, 20, p. 42-44.

AUBIN, G., 2006 - Un trésor d'imitations de Tétricus à Guéhenno (Morbihan). In Trésors de l'Ouest de la France. Trésors monétaires, XXII.Paris, Bibliothèque Nationale de France, p. 249-252.

BARDEL, J.-P., 2007 - L'établissement antique de Plomarc'h Pella à Douarnenez (Finistère) : un ensemble représentatif des établissements de salaison romains de la baie. AREMORICA. études sur l'ouest de la Gaule romaine, 1, p. 101-116.

BATT, M., 2007 - Le Goënidou, Berrien (29): un exemple de parcellaire antique ? AREMORICA. études sur l'ouest de la Gaule romaine, 1, p. 117-122.

BERARD, F., 2006 - Mars Mullo: un Mars des cités occidentales de la province de Lyonnaise, in BROUQUIER-REDDÉ, V. et alii (dir.) - Mars en Occident. (Actes du colloque international "Autour d'Allonnes (Sarthe), les sanctuaires de Mars en Occident", 
Le Mans, Université du Maine, 4-6 juin 2003). Rennes, Presses universitaires de Rennes (coll. Archéologie \& Culture), p. 17-34.

BERDEAUX-LE BRAZIDEC, M.-L., 2006 - Fragments de trésors romains trouvés en Bretagne, conservés au musée d'Archéologie nationale. In Trésors de l'Ouest de la France. Trésors monétaires, XXII. Paris, Bibliothèque Nationale de France, p. 207-248 + 1 pl.

BESOMBES, P.-A. et MORIN, E., 2006 - Le dépôt de la Vilaine à Rennes. In Trésors de l'Ouest de la France. Trésors monétaires, XXII. Paris, Bibliothèque Nationale de France, p. 1-35+ 3 pl.

BIZIEN-JAGLIN, C., 2008 - Le complexe îlien des Ebihens en Saint-Jacut-de-la-Mer. Un aspect de la question de la romanisation chez les Coriosolites. Bulletin de la Société française d'Archéologie classique, 38, $\mathrm{p}$.

CAMBT, C., 2008 - Limites politiques oubliées: Armorique et Bretagne. Critique de sources. Mémoires de la Société d'Histoire et d'Archéologie de Bretagne, 86, p. 89-105.

CHARTIER, E., 2007 - Du nouveau sur les origines de Quimper. ArMen, n 157, p. 59.

CHARTIER, E., 2008 - Une villa gallo-romaine à Ploufragan. ArMen, nº 166, p. 60.

CHEVET, P., 2006 - Le site de la Visitation à Rennes : évolution d'un quartier urbain du $\mathrm{I}^{\mathrm{er}}$ au $\mathrm{IV}^{\mathrm{e}}$ siècle. Bulletin et Mémoires de la Société archéologique d'Ille-et-Vilaine, 110, p. 21-32.

CORRE, A., 2008 - Prospection inventaire en forêt de Rennes (Ille-et-Vilaine). Les Dossiers du CeRAA, 36, p. 45-64

B. et GALLIOU, P., 2007 - Les fouilles du Yaudet en Ploulec'h, Côtes-d'Armor Volume 3 - Du quatrième siècle après J.-C. à aujourd'hui. Oxford, Oxford University School of Archaeology, (Monograph, 65), 207 p.

DARE, S., 2008 - L'établissement antique de la pointe de la Garenne au Hézo (Morbihan). AREMORICA. études sur l'ouest de la Gaule romaine, 2, p. 119-146.

DRIARD, C., 2007 - L'établissement romain de "La Falaise» à Etel. Sondage archéologique d'un atelier de salaisons de poissons. Bulletin de la Société d'Archéologie et d'Histoire du Pays de Lorient, 35 (2006-2007), p. 57-62.

DROST, V., 2006 - Le trésor de la préfecture de Rennes (Ille-et-Vilaine), 1881. In Trésors de l'Ouest de la France. Trésors monétaires, XXII. Paris, Bibliothèque Nationale de France, p. $171-206+4 \mathrm{pl}$.

ERISTOV, H., GROETEMBRIL, S., 2006 - Murs blancs en Gaule. Entre économie et raffinement.

Dossiers d'Archéologie, n 318, p. 58-61.

EVEILLARD, J.-Y., 2006 - Une statuette en bronze de l'Epona (?) gallo-romaine dans le Sud-Finistère. Bulletin de la Société archéologique du Finistère, 135, p. 29-36.

VEILLARD, J.-Y., 2006 - Douarnenez et Corseul : analyse comparative de deux statues masculines en granite. Patrimoine, bulletin de la Société archéologique de Corseul, 20, p. 28-30. EVEILLARD, J.-Y., 2007 - Actualité de la sculpture en pierre d'époque romaine en Bretagne. AREMORICA. études sur l'ouest de la Gaule romaine, 1, p. 123-129.

EVEILLARD, J.-Y. et BARDEL, J.-P., 2007 - Le site des Plomarc'h en Douarnenez (Finistère) : un modèle pour le fonctionnement des usines de salaisons sur la façade nord-ouest atlantique? In LAGOSTENA, L., BERNAL, D. et ARÉVALO, A. (dir.) - Salsas y 
Salazones de Pescado en Occidente durante la Antigüedad? (Actas del Congreso internacional deCàdiz, 7-9 nov. 2005). Oxford, Archaeopress, (BAR International Series 1686), p. 151-156.

EVEILLARD, J.-Y., 2008 - Graffite et portrait d'un Osisme sur une plaquette de schiste à Brasparts (Finistère). AREMORICA. études sur l'ouest de la Gaule romaine, 2, p.105-117.

EVEILLARD, J.-Y., 2008 - Les voies romaines en Bretagne. L'Archéologue - Archéologie nouvelle, $\mathrm{n}^{\circ} 98, \mathrm{p} .32-35$.

EVEILLARD, J.-Y., 2008 - A propos de la découverte d'une statue de Neptune à Douarnenez (Finistère) : Caius Varenius Varus, producteur de salaisons de poissons, in Ressources et activités marines des peuples de l'Antiquité. (Actes du colloque international de Boulogne-sur-Mer 12-14 mai 2005). Boulogne-sur-Mer, Centre de Recherche en Histoire atlantique et littorale, (Les cahiers du littoral, 2-6), p. 395-400.

310 FERRETTE, R., 2006 - Découverte d'un temple de tradition celtique à la sortie est du bourg de Corseul. Patrimoine, bulletin de la Société archéologique de Corseul, 20, p. 17-22.

FERRETTE, R., 2006 - Villa en Côtes-d'Armor. L 'Archéologue - Archéologie nouvelle, 84, p. 48-49.

312 FERRETTE, R., 2007 - La villa des Alleux, premiers bilans de l'opération archéologique. Patrimoine, bulletin de la Société archéologique de Corseul, 21, p. 12-19.

313 FERRETTE, R., 2007 - Notions sur la production et l'apport de la céramique en archéologie. Patrimoine, bulletin de la Société archéologique de Corseul, 21, p. 33-39. FERRETTE, R., 2007 - Un établissement rural gallo-romain sur la commune de Plouërsur-Rance (Côtes-d'Armor). Revue archéologique de l'Ouest, 24, p. 121-138. FEUGÈRE, M., 2008 - Le dépôt d'objets métalliques de la rue de Saint-Malo à Rennes, in POUILLE, D., Rennes antique. Rennes, Presses universitaires de Rennes (coll. Archéologie \& Patrimoine), p. 361-372.

316 GARBARINI, J., HELAUDAIS, J.-F. et BIZIEN-JAGLIN, C., 2006 - Un nouveau lot de monnaies antiques à Alet et quelques objets divers. Les Dossiers du CeRAA, 34, p. 9-19.

317 GALLIOU, P., 2008 - L'habitat rural de l'Armorique romaine, in TONNERRE N.-Y. (dir.) La maison paysanne en Bretagne : 2500 ans d'habitat rural. Spézet, Coop Breizh, p. 16-27. GUIRAUD, H., 2008 - Intaille ${ }^{\circ} 1236$ - Corseul, in GUIRAUD, H. - Intailles et camées de l'époque romaine en Gaule. (vol. II). Gallia., suppl.48, p. 127. GUIRAUD, H., 2008 - Intaille $\mathrm{n}^{\circ} 1300$ - Rennes, in GUIRAUD, H. - Intailles et camées de l'époque romaine en Gaule. (vol. II). Gallia., suppl.48, p. 145. GUIRAUD, H., 2008 - Intaille $\mathrm{n}^{\circ} 1275$ - Corseul, in GUIRAUD, H. - Intailles et camées de l'époque romaine en Gaule. (vol. II). Gallia., suppl.48, p. 140. GUIRAUD, H., 2008 - Intaille ${ }^{\circ} 1415$ - Corseul, in GUIRAUD, H. - Intailles et camées de l'époque romaine en Gaule. (vol. II). Gallia., suppl.48, p. 141. GUIRAUD, H., 2008 - Intaille $\mathrm{n}^{\circ} 1373$ - Corseul, in GUIRAUD, H. - Intailles et camées de l'époque romaine en Gaule. (vol. II). Gallia., suppl.48, p. 160. GUIRAUD, H, 2008 - Intaille ${ }^{\circ} 1334$ - Quimper, in GUIRAUD, H. - Intailles et camées de l'époque romaine en Gaule. (vol. II). Gallia., suppl.48, p. 153. 
HOLLARD, D. et LE FLOC'H, Y., 2006 - Le trésor de Pédernec (Côtes-d'Armor). In Trésors de l'Ouest de la France. Trésors monétaires, XXII. Paris, Bibliothèque Nationale de France, p. $133-169+10 \mathrm{pl}$.

LABAUNE-JEAN, F., 2008 - Le travail de l'os à Rennes (Ille-et-Vilaine) à travers un canif à manche sculpté trouvé 3-5 rue de Saint-Malo, 2008, in BERTRAND, I. (dir.) - Le travail de l'os, du bois de cerf et de la corne à l'époque romaine, un artisanat en marge? Montagnac, éd. Monique Mergoil, (monographies Instrumentum, 34), p. 55-63.

LABAUNE-JEAN, F. et LE CLOIREC, G., 2008 - Le verre antique de Carhaix (Finistère), chef-lieu des Osismes. Bulletin de l'Association française pour l'Archéologie du Verre, p. 24-29.

LABAUNE-JEAN, F. et PROVOST, A., 2008 - La verrerie antique de la villa de ManéVéchen à Plouhinec (Morbihan). Bulletin de l'Association française pour l'Archéologie du Verre, p. 30-35.

LABAUNE-JEAN, F., 2008 - Les enduits peints en Bretagne gallo-romaine, état des dernières découvertes. AREMORICA. études sur l'ouest de la Gaule romaine, 2, p. 9-42

LABAUNE-JEAN, F., 2008 - Une pièce exceptionnelle: le plat de type Isings 97c de Corseul. Patrimoine, bulletin de la Société archéologique de Corseul, 22, p. 3-5.

LABAUNE-JEAN, F., 2008 - Graffiti Rennais : Abécédaire gravé, in BARBET, A. et FUCHS, M. (dir.) - Les murs murmurent: graffitis gallo-romains. Gollion (Suisse), éd. Infolio, p. 53-56 et 61-62.

LABAUNE-JEAN, F., 2008 - Graffiti Rennais : Cheval au pas, in BARBET, A. et FUCHS, M. (dir.) - Les murs murmurent : graffitis gallo-romains. Gollion (Suisse), éd. Infolio, p. 107-108 et 124-125.

LABAUNE-JEAN, F., 2008 - Graffiti Rennais : Amour et injures, in BARBET, A. et FUCHS, M. (dir.) - Les murs murmurent : graffitis gallo-romains. Gollion (Suisse), éd. Infolio, p. 142 et $148-149$.

LABAUNE-JEAN, F., 2008 - Graffiti Rennais : Vive Quintus Murranus, in BARBET, A. et FUCHS, M. (dir.) - Les murs murmurent : graffitis gallo-romains. Gollion (Suisse), éd. Infolio, p. 173-174 et 191.

4 LABAUNE-JEAN, F., 2008 - Le verre, in LE CLOIREC, G. - Carhaix antique : la domus $d u$ centre hospitalier. Contribution à l'histoire de Vorgium? chef-lieu de la cité des Osismes. Rennes, Presses universitaires de Rennes (coll. Documents archéologiques), p. 143-151.

5 LABAUNE-JEAN, F., 2008 - Datations fournies par le mobilier du Campus Hoche, in POUILLE, D. (dir), Rennes antique. Rennes, Presses universitaires de Rennes, (coll. Archéologie \& Culture), p. 118-160.

6 LABAUNE-JEAN, F., 2008 - Parking Hoche : Datations et étude du mobilier, in POUILLE, D. (dir) - Rennes antique. Rennes, Presses universitaires de Rennes, (coll. Archéologie \& Culture), p. 210-249.

LANGOUËT, L., 2006 - Le Portus Saliocanus était-il au pied du Yaudet à Ploulec'h (Côtesd'Armor) ? Les Dossiers du CeRAA, 34, p. 21-27.

LANGOUËT, L., 2006 - Réflexion sur les sites portuaires de la Civitas des Coriosolites. Patrimoine, bulletin de la Société archéologique de Corseul, 20, p. 23-27.

LE BIHAN, J.-P., 2007 - Les cultes antiques à Ouessant. Deux millénaires d'étranges pratiques religieuses. ArMen, $\mathrm{n}^{\circ}$ 160, p. 6-15. 
LE BIHAN, J.-P., 2007 - Quimper-Locmaria antique. Une genèse et une chronologie encore complexes. Archéopages, $\mathrm{n}^{\circ}$ 20, p. 38-43.

LE BOHEC, Y., 2008 - La province romaine de Gaule Lyonnaise (Gallia Lugudunensis), du Lyonnais au Finistère. Dijon, éd. Faton, 358 p.

LE BOULANGER, F. et SIMON, L., 2008 - Les objets en verre de la nécropole de SaintMarcel (Morbihan). Bulletin de l'Association française pour l'Archéologie du Verre, p. 78-81.

LE BOUTEILLER, R., 2006 - A propos de Corseul: une étude sur les Curiosolites. Association Bretonne, 114, p. 45-59.

CLOIREC, G., 2007 - La voirie urbaine des chefs-lieux de la Bretagne romaine. Réfexions à partir d'exemples fouillés récemment. AREMORICA. études sur l'ouest de la Gaule romaine, 1, p. 39-54.

LE CLOIREC, G., 2007 - Carhaix, ville romaine. Patrimoine, bulletin de la Société archéologique de Corseul, 21, p. 8-11.

LE CLOIREC, G. et POUILLE, D., 2007 - Les chefs-lieux de civitas de la péninsule armoricaine : Condate, Darioritum, Fanum Martis, Vorgium, quatre villes fortement éprises des modèles romains. Archéopages, $\mathrm{n}^{\circ}$ 20, p. 32-37.

LE CLOIREC, G. (dir.), 2008 - Carhaix antique : la domus du centre hospitalier. Contribution à l'histoire de Vorgium, chef-lieu de la cité des Osismes. Rennes, Presses universitaires de Rennes, (coll. Documents archéologiques), $263 \mathrm{p}$.

LE CLOIREC, G., 2008 - La voirie des chefs-lieux gallo-romains de la péninsule armoricaine, in BALLET, P., DIEUDONNÉ-GLAD, N. ET SALIOU, C. (dir.) - La rue dans l'Antiquité. Définition, aménagement et devenir, de l'Orient méditerranéen à la Gaule. Rennes, Presses universitaires de Rennes (coll. Archéologie \& Culture), p. 321-325.

LECORNEC, J. et SAUJOT-BESNIER, C.,2006 - Ossaria ou pile-mil : le débat n'est pas clos. Revue archéologique de l'Ouest, 23, p. 199-201.

LEPETZ, S., POUILLE, D. et LABAUNE-JEAN, F., 2008 - Les dépôts alimentaires de RennesCondate, témoignages de rituels célébrés en contexte privé ? In LEPETZ, S. et VAN ANDRINGA, W. (dir.) - Archéologie du sacrifice animal en Gaule romaine: rituels et pratiques alimentaires. Montagnac, éd. Monique Mergoil, (coll. Archéologie des plantes et des animaux, 2), p. 125-136.

LEROUX, G., 2007 - Une section de la voie antique Vannes-Angers. Archéopages, $\mathrm{n}^{\circ} 18$, p. 74-75.

MALIGORNE, Y., 2006 - Le décor architectonique de Corseul et de la civitas des Coriosolites. Patrimoine, bulletin de la Société archéologique de Corseul, 20, p. 31-37. MALIGORNE, Y., 2006 - L'architecture romaine dans l'ouest de la Gaule. Rennes, Presses universitaires de Rennes (coll. Archéologie \& Culture), 229 p. siècles.

Patrimoine, bulletin de la Société archéologique de Corseul, 21, p. 3-7.

MALIGORNE, Y., 2007 - Sanctuaires et structures vicinales dans deux chefs-lieux de l'ouest de la Gaule. A propos de quatre inscriptions de Nantes et Angers. AREMORICA. études sur l'ouest de la Gaule romaine, 1, p. 57-71. 
Revue archéologique de l'Ouest, 26 | 2009 p. 399-418. romaine, 2, p. 155-203. Archéologie \& Culture), $438 \mathrm{p}$. 100), $477 \mathrm{p}$. \& Culture), p. 373-390. 2006. Marseille, SFECAG, p. 673-676.

MALIGORNE, Y., 2008 - Bono reipublicae natus: une louange impériale sur quelques monuments de l'Antiquité tardive (à propos d'une inscription de Lancieux : CIL.- XIII.8994=XVII-2.- 420a). Revue archéologique de l'Ouest, 25, p. 291-304.

MATTHIEU, N., 2006 - L'époque gallo-romaine, In Bretagne est univers. Catalogue du Musée de Bretagne. Rennes, co-éd. Apogée - Presses universitaires de Rennes, p. 55-71.

MONTEMBAULT, V., 2008 - Le cuir, in LE CLOIREC, G. - Carhaix antique : la domus $d u$ Centre hospitalier. Contribution à l'histoire de Vorgium, chef-lieu de la cité des Osismes. Rennes, Presses universitaires de Rennes (coll. Documents archéologiques), p. 158-162.

NAAS, P., 2008 - Les enclos d'époque romaine en Armorique pénisulaire et dans les régions limitrophes. AREMORICA. études sur l'ouest de la Gaule romaine, 2, p. 67-85.

PERNOT, M. et MOTHES, C., 2008 - Étude technologique d'un échantillon de mobiliers issus des ateliers de travail des métaux et alliages non ferreux, in POUILLE, D. - Rennes antique. Rennes, Presses universitaires de Rennes (coll. Archéologie \& Culture),

PERRIN, M.-Y., 2006 - La fabrique d'une cité dans l'antiquité tardive. Patrimoine, bulletin de la Société archéologique de Corseul, 20, p. 9-16.

PHILIPPE, E., PROVOST, A. et LEPRÊTRE, B., 2008 - L'aqueduc inachevé de l'agglomération antique de Locmariaquer. AREMORICA. études sur l'ouest de la Gaule

POUILLE, D., 2008 - La muraille antique de Rennes. Quelques aspects de la partie sud. AREMORICA. études sur l'ouest de la Gaule romaine, 2, p. 43-66.

POUILLE, D. (dir), 2008 - Rennes antique. Rennes, Presses universitaires de Rennes (coll.

PROVOST, A., 2007 - La «villa» maritime de Mané-Véchen à Plouhinec (Morbihan). AREMORICA. études sur l'ouest de la Gaule romaine, 1, p. 85-100.

REDDE, M., BRULET, R., FELLMANN, R., HAALEBOS, J.-K. et SCHNURBEIN, S. von, 2006 Les fortifications militaires. Paris, éditions de la Maison des Sciences de l'Homme, (DAF,

SERNEELS, V., 2008 - Les activités métallurgiques sur le site de la place Hoche, in POUILLE, D., Rennes antique. Rennes, Presses universitaires de Rennes (coll. Archéologie

SIMON, L., 2006 - La nécropole gallo-romaine de Chantepie (Ille-et-Vilaine), in Actes du Congrès de la SFECAG, Pezenas, 25-28 mai 2006. Marseille, SFECAG, p. 677-679.

SIMON, L., 2006 - Mobiliers en usage chez les Riedones au cours du Haut-Empire: l'exemple de Gévezé (Ille-et-Vilaine), in Actes du Congrès de la SFECAG, Pezenas, 25- 28 mai

SIMON, L. et BERARD, F., 2007 - Conteneurs et contenus : à propos d'un "vase à bière " et du mobilier amphorique du site de Bilaire à Vannes (Morbihan), in Actes du Congrès de la SFECAG, Langres, 17-20 mai 2007. Marseille, SFECAG, p. 595-600.

SIMON, L., 2008 - Enquête en cours sur la verrerie en usage à Vannes durant la période gallo-romaine. Bulletin de l'Association française pour l'Archéologie du Verre, p. 67-68.

SIMON, L., 2008 - De nouvelles productions dans le « Quoit Brooch Style» mises au jour en Bretagne : les découvertes de Saint-Marcel (Morbihan), Instrumentum, n 28, p. 28-29. 

l'Archéologie du Verre, p. 20-23.

\section{9. Époque médiévale} Bretagne, 85, p. 40-56. 19), p. 23-46. Coop Breizh, p. 68-77.

TRISTE, A., 2008 - L'atelier de verriers antique de Kerfloc'h à Plaudren (Morbihan). AREMORICA. études sur l'ouest de la Gaule romaine, 2, p. 87-103.

TRISTE, A. et DARE, S., 2008 - L'atelier de potiers gallo-romain de Liscorno (Morbihan). Bulletin et Mémoires de la Société polymathique du Morbihan, 134, p. 7- 44.

VIVET, J.-B., 2008 - Pilleverte II en Plesder (35) : un atelier armoricain de production massive de fer au Haut-Empire. Les Dossiers du CeRAA, 36, p. 73-102.

TRISTE, A. et DARE, S., 2006 - Découverte d'un nouvel atelier de verriers antique en Bretagne: le site de Kerfloc'h à Plaudren (Morbihan), in 20e Rencontres de l'AFAV, Bavay et Sars-Poteries, 13-14 octobre 2005. Bulletin de l'Association française pour

AQUILINA, M., 2007 - «Murs de ville" à Quimper, «fortifications" à Concarneau : destins croisés de remparts médiévaux. Mémoires de la Société d'Histoire et d'Archéologie de

BARDEL, A. et PERENNEC. R., 2006 - Les cuisines et le réfectoire de l'abbaye de Landévennec (Finistère). in Production alimentaire et lieux de consommation dans les établissements religieux au Moyen Âge et à l'époque moderne - Tome 1. (Colloque du CAHMER, Lille, 16-18 octobre 2003). Amiens, CAHMER (coll. Histoire médiévale et Archéologie,

BATT, M., 2008 - La maison paysanne du Moyen Âge en Bretagne: apport de l'archéologie et comparaisons avec le sud-ouest de l'Angleterre et le Pays de Galles, in TONNERRE, N.-Y. (dir.) - La maison paysanne en Bretagne : 2500 ans d'habitat rural. Spézet,

BERGER, C. et SALLOU, F., 2007 - Relevés de la motte féodale de Coz Castel en Plounez. ARSSAT - Bulletin annuel, 32, p. 104-117.

BEUCHET, L. et MARTINEAU, J., 2008 - Archéologie préventive des châteaux de la Bretagne Ducale: résultats récents et perspectives de recherches, in Bilan des recherches en castellologie (colloqueInternational de Houffalize (Belgique), 4-10 septembre 2006). Château-Gaillard, 23, p. 33-44.

BIENVENU, J., 2008 - Des fortifications à Plancoët (22) à la fin du XIVème siècle ? De nouvelles données. Les Dossiers du CeRAA, 36, p. 5-19.

BRANDILY, D., 2007 - Des quais anciens découverts à Mordreuc (Pleudihen-sur-Rance, Côtes-d'Armor). Les Dossiers du CeRAA, 35, p. 73-80.

CHARTIER, E., 2007 - Archéologie expérimentale à Melrand. ArMen, n 156, p. 60-61.

CHARTIER, E., 2007 - Le château du Guildo. ArMen, n 158, p. 59.

CHARTIER, E., 2008 - Le château de La Hunaudaye. ArMen, n 164, p. 34-39.

CHAURIS, L, 2008 - La pierre dans les constructions à Lannion. Mémoires de la Société d'Histoire et d'Archéologie de Bretagne, 86, p. 5-25.

CLAIRAND, A., 2006 - Le trésor de Neuillac (Morbihan) : 117 monnaies d'or du XIv siècle, in Trésors de l'Ouest de la France. Trésors monétaires, XXII. Paris, Bibliothèque Nationale de France, 22, p. 253-267 + 2 pl. 
COATIVY, Y., 2006 - La monnaie des ducs de Bretagne, de l'an mil à 1499. Rennes, Presses universitaires de Rennes, $454 \mathrm{p}$.

COATIVY, Y., 2008 - L'atelier monétaire de Vannes au Moyen Âge. Bulletin et Mémoires de la Société Polymathique du Morbihan, 134, p. 73-77.

COLLETER, R., JEAN, S., LE BOULANGER, F. et PICHOT, D., 2006 - Bréal-sous-Vitré (Illeet-Vilaine) : du cimetière du haut Moyen Âge à la paroisse. Mémoires de la Société d'Histoire et d'Archéologie de Bretagne, 84, (Congrès de Vitré, 8-10 septembre 2005), p. 531-556.

CONTE, P. et BATT, M., 2006 - "Maison-mixte " et bâtiments à plan absidial en Bretagne et dans le Limousin du XI ${ }^{\mathrm{e}}$ au XVI ${ }^{\mathrm{e}}$ siècle, in Cadre de vie et manières d'habiter. (8e congrès international de la Société d'Archéologie médiévale, Paris, 11-13 octobre 2001). Caen, CRAHM, p. 21-38.

CRINON, P., 2008 - Nouvelle datation du trésor de Bais, à propos des deniers épiscopaux de Sens. Bulletin de la Société française de Numismatique, 63e année, 06, p. 82-93.

CUCARULL, J., 2007 - Destruction symbolique et persistance emblématique d'un château médiéval, Saint-Aubin-du-Cormier (1490-2004), in La forteresse à l'épreuve du temps. Destruction, dissolution, dénaturation, Xle-XXe siècle (129e congrès national des Sociétés savantes, Besançon, 2004). Paris, Comité des Travaux Historiques et Scientifiques (Archéologie et Histoire de l'Art, 26), p. 51-61.

DEBIAIS, V., 2008 - Corpus des inscriptions de la France médiévale. Volume 23: Côtesd'Armor, Finistère, Ille-et-Vilaine, Morbihan (région Bretagne), Loire-Atlantique et Vendée (région Pays de la Loire). Paris, CNRS, (Corpus des inscriptions de la France médiévale), $164 \mathrm{p}$.

397 FICHET de CLAIRFONTAINE, F. et DUFOURNIER, D., 2006 - La maison du potier, du XI ${ }^{\mathrm{e}}$ au XVII ${ }^{e}$ siècle dans la France du nord-ouest, in ALEXANDRE-BIDON, D., PIPONNIER, F. et POISSON, J.-M. (dir.) - Cadre de vie et manières d'habiter. Caen, CRAHM, p. 267-273.

GUIGON, P. et MEURET, J.-C., 2006 - La réutilisation de sarcophages dans les églises de l'est de la Bretagne. Mémoires de la Société d'Histoire et d'Archéologie de Bretagne, 84 (Congrès de Vitré, 8-10 septembre 2005), p. 355-405.

JONES, M., MEIRION-JONES, G. et GUIBAL, F., 2008 - Coadélan en Prat, Côtes-d'Armor. Mémoires de la Société d'Histoire et d'Archéologie de Bretagne, 86, p. 437-477.

KERNEVEZ, P., 2007 - La Ville Close de Concarneau. Mémoires de la Société d'Histoire et d'Archéologie de Bretagne, 85, p. 467-471.

KERNEVEZ, P., 2008 - Le château de Tonquédec. Mémoires de la Société d'Histoire et d'Archéologie de Bretagne, 86, p. 431-436.

LABAUNE-JEAN, F. et LE BOULANGER, F., 2006 - Les objets de parure de Visseiche (Illeet-Vilaine) (20e Rencontres de l'AFAV, Bavay et Sars-Poteries, 13-14 octobre 2005). Bulletin de l'Association française pour l'Archéologie du Verre, p. 18-19.

LABAUNE-JEAN, F. et BEUCHET, L., 2008 - Le château du Guildo à Créhen (Côtesd'Armor). Les pièces de verrerie. Bulletin de l'Association française pour l'Archéologie du Verre, p. 97-102.

LANGOUËT, L., 2006 - La sépulture viking à barque de l'île de Groix (Morbihan). Bulletin d'information de l'AMARAI, 19, p. 87-108. 
LE BOULANGER, F., 2007 - Naissance d'une paroisse revue grâce à l'archéologie et à l'anthropologie. Archéopages, n 19, p. 78-79.

LE GALL-TANGUY, R., 2006 - Morphogenèse de la ville de Carhaix au Moyen Âge. Bulletin de la Société archéologique du Finistère, 135, p. 71-91.

407 LE GOFF, J.-Y., 2007 - Châteaux et manoirs du canton de Taulé. Quimper, Société finistérienne d'Histoire et d'Archéologie, $145 \mathrm{p}$.

408 LEROY, B., 2008 - Les monnayages mérovingiens armoricains. Paris, Association française d'Archéologie mérovingienne (Bulletin de liaison, hors-série 3), $126 \mathrm{p}$.

409 LE QUELLEC, V., LE GOFFIC, M. et PEUZIAT, J., 2006 - Les enceintes médiévales du Porzay (Finistère). Bulletin de la Société archéologique du Finistère, 135, p. 37-69.

410 LETTRY-HERIO, H., 2007 - Le château de Keroman. Bulletin de la Société d'Archéologie et d'Histoire du Pays de Lorient, 35 (2006-2007), p. 149-152.

411 MARTINEAU, J., 2008 - Trésor de guerre. Histoire et Images médiévales. Thématique, 14,p. 34-35.

412 PICHOT, D., 2008 - La maison et la société en Bretagne ( $\mathrm{xl}^{\mathrm{e}}$-XIII ${ }^{\mathrm{e}}$ siècle), in TONNERRE, N.Y. (dir.) - La maison paysanne en Bretagne: 2500 ans d'habitat rural. Spézet, Coop Breizh, p. 52-67.

413 TONNERRE, N.-Y., 2008 - L'habitat rural du haut Moyen Âge: une réévaluation indispensable, in TONNERRE, N.-Y. (dir.) - La maison paysanne en Bretagne: 2500 ans d'habitat rural. Spézet, Coop Breizh, p. 28-35.

414 TOURNIER, F., 2007 - Étude archéologique au couvent des Jacobins. Le Pays de Dinan, $\mathrm{n}^{\circ} 27$, n.p.

415 TRISTE, A. et DARE, S., 2006 - Fouilles archéologiques sur le site de la chapelle de Mangolérian à Monterblanc. Bulletin et Mémoires de la société polymathique du Morbihan, 132, p. 55-67.

416 TRISTE, A. et DARE, S., 2008 - Carmes et carmélites du Bondon (Vannes) : découverte des vestiges des couvents. Bulletin d'information du Céram, 2, p. 6-19.

\section{0. Époque moderne et contemporaine}

417 CHARTIER, E., 2006 - Vauban en Bretagne. Un patrimoine à mettre en valeur. ArMen, $\mathrm{n}^{\circ} 160$, p. 22-31.

418 CHAURIS, L., 2006 - Éclairage lthologique sur l'église de Lanhouarneau (Finistère : XIV e$\mathrm{XVI}^{\mathrm{e}}$-XVIII ${ }^{\mathrm{e}}$ siècles). Revue archéologique de l'Ouest, 23, p. 137-149.

DONGUY, J.-R. et PHILIPPE, J.-P., 2007 - L'épave de Trez-Malouen. Le Chasse-Marée, $\mathrm{n}^{\circ} 201$, p. 33-41.

420 GUILLOU, M., 2007 - Les fortifications du Mur de l'Atlantique en Bretagne nord. ARSSAT - Bulletin annuel, 32, p. 37-41

421 LE CAM, G., 2007 - le village de poul-Fétan, joyau de l'architecture rurale ( $\mathrm{Xv}^{\mathrm{e}}$ XVII ${ }^{e}$ siècles). Bulletin de la Société d'Archéologie et d'Histoire du Pays de Lorient, 35 (2006-2007), p. 105-108. 
LE CAM, G., 2007 - L'usine à fer de Kerglaw sur le Blavet, cent ans de vie ouvrière aux forges d'Hennebont. Bulletin de la Société d'Archéologie et d'Histoire du Pays de Lorient, 35 (2006-2007), p. 201-210.

LE COLLETER, C., 2007 - La redoute-batterie de Kerhonno-Lanester. Bulletin de la Société d'Archéologie et d'Histoire du Pays de Lorient, 35 (2006-2007), p. 115-117.

PENVEN, L., 2008 - Le fort de Keragan dit Fort Bloqué. Bulletin du Comité d'Histoire du Pays de Ploemeur, 18, p. 2-13.

POPE, P. et BATT, M., 2008 - Post-medieval Breton earthenwares in Newfoundland. PostMedieval Archaeology, 42 (1), p. 48-74.

SCUILLER, S., 2008 - Propriété et usages collectifs. L'exemple des marais de Redon au $\mathrm{XVIII}^{\mathrm{e}}$ siècle. Histoire et Sociétés Rurales, 29, p. 41-71.

OURNIER, F., 2006 - Un bâtiment mauriste (XVII siècle) retrouvé à l'abbaye de SaintMathieu (Finistère). Revue archéologique de l'Ouest, 23, p. 71-86. VASSART, R., 2008 - La défense fixe des côtes dans le passé de Larmor-Plage : le témoin caché de Toulhars. Bulletin du Comité d'Histoire du Pays de Ploemeur, 18, p. 19-28.

\section{AUTEURS}

\section{MARIE-DOMINIQUE PINEL}

Service régional de l'Archéologie, DRAC de Bretagne : 6 rue du Chapitre, 35044 Rennes cedex CATHERINE LE GALL

CNRS, UMR 6566 - CreAAH : Université de Rennes 1, campus de Beaulieu, Bât 24-25, CS 74205, 35042 Rennes cedex 